\title{
Nuclear starburst-driven evolution of the central region in NGC 6764
}

\author{
S. Leon ${ }^{1}$, A. Eckart ${ }^{2}$, S. Laine ${ }^{3}$, J. K. Kotilainen ${ }^{4}$, E. Schinnerer ${ }^{5}$, \\ S.-W. Lee ${ }^{6}$, M. Krips ${ }^{2}$, J. Reunanen ${ }^{7}$, and J. Scharwächter ${ }^{8}$ \\ 1 Instituto de Radio Astronomía Milimétrica, Granada, Spain \\ e-mail: leon@iram.es \\ 2 University of Cologne, I. Physikalisches Institut, Cologne, Germany \\ Spitzer Science Center, Caltech, Pasadena, USA \\ Tuorla Observatory, University of Turku, Piikkiö, Finland \\ Max-Planck-Institut für Astronomie, Heidelberg, Germany \\ ${ }^{6}$ University of Toronto, Astronomy Department, Toronto, Canada \\ 7 University of Leiden, Department of Astronomy, Leiden, The Netherlands \\ ${ }^{8}$ European Southern Observatory, Santiago, Chile
}

Received 20 July 2006 / Accepted 29 June 2007

\section{ABSTRACT}

\begin{abstract}
Aims. We study the $\mathrm{CO}$ and the radio continuum emission in an active galaxy to analyze the interplay between the central activity and the molecular gas.

Methods. We present new high-resolution observations of the ${ }^{12} \mathrm{CO}(1-0)$ and ${ }^{12} \mathrm{CO}(2-1)$ emission lines, and $3.5 \mathrm{~cm}$ and $20 \mathrm{~cm}$ radio continuum emission in the central region of the LINER/starburst galaxy NGC 6764

Results. The galaxy has an outflow morphology in radio continuum, spatially coincident with the $\mathrm{CO}$ and $\mathrm{H} \alpha$ emission, and centered slightly off the radio continuum peak at the LINER nucleus. The total molecular gas mass in the center is about $7 \times 10^{8} \mathcal{M}_{\odot}$, using a CO luminosity to total molecular gas conversion factor that is three times lower than the standard one. ${ }^{12} \mathrm{CO}(1-0)$ emission is found near the boundaries of the radio continuum emission cone. The outflow has a projected expansion velocity of $25 \mathrm{~km} \mathrm{~s}^{-1}$ relative to the systemic velocity of NGC 6764 . About $4 \times 10^{6} \mathcal{M}_{\odot}$ of molecular gas is detected in the outflow. The approximate location $(\sim 1 \mathrm{kpc})$ of the dynamical inner Lindblad resonance has been derived from the rotation curve. The peak of the CO emission is slightly $(<200 \mathrm{pc})$ offset from the peak of the radio continuum.

Conclusions. The molecular gas has most likely been ejected by the stellar winds from the recent starburst, but the $\mathrm{CO}$ line ratios show indication of an interaction with the AGN. The energy released by the nuclear starburst is sufficient to explain the observed outflow, even if the data cannot exclude the AGN from being the major energy source. Comparison of the outflow with hydrodynamical simulations suggests that the nuclear starburst is 3-7 Myr old and the bubble-like outflow is still confined and not freely expanding.
\end{abstract}

Key words. ISM: jets and outflows - galaxies: active - ISM: kinematics and dynamics - ISM: individual: objects: NGC 6764 radio lines: galaxies

\section{Introduction}

The nuclear region surrounding active galactic nuclei (AGN) often has the highest level of molecular gas concentration and star formation in galaxies. The processes that drive the excitation and kinematics of the circumnuclear gas, namely, the abundant star formation and the jets and photoionisation associated with the AGN, influence the circumnuclear gas in several ways. The onset and shutdown of the starburst are driven by the properties of the molecular gas, including its density and velocity dispersion, by gravitational instabilities (e.g., Combes 2001), and by processes that tend to prevent star formation, such as the local tidal field (Launhardt et al. 2002), starburst feedback (e.g., Dopita 1985; Scalo \& Chapell 1999), gas consumption (e.g., Franceschini et al. 1998), and tidal shear (e.g., Kenney et al. 1993). Fueling of starbursts is facilitated by nonaxisymmetric components such as bars, and by tidal torques from interactions and mergers (e.g. Combes \& Gerin 1985; Athanassoula 1992; Mihos \& Hernquist 1994; Laine et al. 2002)
The formation of powerful gas and plasma outflows from the center of disk galaxies provides the basis for the metal enrichment of galaxy halos (e.g., Kunth et al. 2002). Several mechanisms have been proposed to explain these outflows. The first possibility is a thermal wind from a circumnuclear starburst (e.g., Colina et al. 1999). The second possibility is a thermal wind from an AGN, as suggested by, e.g., Krolik \& Bergelman (1986). Finally, it is possible that ram-pressure along the radio jet is driving the outflow (e.g., Taylor et al. 1992). Large-scale radio halos outside the galaxy disk, which most likely result from plasma and magnetic fields that have been blown off the disk by one of the above-mentioned mechanisms, have been known for more than two decades (e.g., Hummel et al. 1983; Hummel et al. 1991).

The nuclear starburst leads to the formation of OB associations or super star clusters, from which the massive stars, through strong supersonic stellar winds, inject both energy and mass into the surrounding interstellar medium. The formation of an expanding starburst "superbubble" will be accompanied 
by extended X-ray emission from hot gas, and optical $\mathrm{H} \alpha$ line emission from the cooler shell. The superbubble that has a larger internal ISM pressure than its surroundings expands into the low-pressure halo ISM until the onset of Rayleigh-Taylor instabilities, which disrupt the superbubble and allow the hot gas to escape in the form of a galactic wind, as is seen, e.g., in M 82 (Strickland et al. 1997). The cold molecular gas is expected to be compressed around the superbubble into an expanding cocoon.

Molecular gas in outflows has been detected in a few galaxies. M 82 has an outflow bubble expanding at $45 \mathrm{~km} \mathrm{~s}^{-1}$ (Weiß et al. 1999), with a molecular outflow gas mass of $\sim 10^{6} \mathcal{M}_{\odot}$. NGC 2782 harbors an outflow with about $\sim 2 \times 10^{7} \mathcal{M}_{\odot}$ of molecular gas (Jogee et al. 1998). The outflow in NGC 2782 appears to be in the early stages (its age is $\sim 4 \times 10^{6}$ years), so that it is seen before the onset of Rayleigh-Taylor instabilities. In NGC 3079 four expanding CO shells have been identified (Irwin \& Sofue 1996). These appear to have originated from a single starburst that took place a few Myrs ago.

The behavior of the molecular gas in such energetic outflows is still poorly understood. The kinematic and potential energies of the molecular gas are part of the total energy budget of the outflow, and one can use the observed properties of the gas (its location, mass and velocity) to constrain the origin of the outflow. Recently Sakamoto et al. (2006) observed molecular gas shells with the Submillimeter Array (SMA) in the circumnuclear disk of NGC 253. Further constraints on the nature and location of the energy source can be provided by the current gas phase, as in NGC 2782 (Jogee et al. 1998), or an unusual geometry of the outflow, such as the off-center outflow in NGC 5775 (Lee et al. 2001). Since gas is fuel for star formation, when it falls back onto the galaxy disk it may induce new episodes of star formation (e.g., Norman \& Ikeuchi 1989).

NGC 6764 is a barred spiral galaxy with an optically classified low-ionisation nuclear emission-line region (LINER) nucleus (Osterbrock \& Cohen 1982). This nearby active galaxy $\left(D=32 \mathrm{Mpc} ; 1^{\prime \prime}=160 \mathrm{pc}\right)$ hosts a very young nuclear starburst as shown by its Wolf-Rayet (W-R) feature at $0.47 \mu \mathrm{m}$ (HeII; Schinnerer et al. 2000). Eckart et al. (1991, 1996) and Schinnerer et al. (2000) analyzed the central starburst, traced by the W-R emission, in great detail. The millimetre observations have revealed a central concentration of molecular gas which also emits near-infrared line emission. The $2.12 \mu \mathrm{m} \mathrm{H}_{2}$ line has features that are also seen in radio continuum. A population synthesis model based on NIR spectral lines suggests that NGC 6764 has undergone two recent starbursts. One of them took place 3-5 Myr ago, producing W-R features, and another 15-50 Myr ago. ROSAT X-ray data trace mainly the central AGN which varies by a factor of two over seven days (Schinnerer et al. 2000). By studying $\mathrm{HCN}$ and $\mathrm{CO}$ emission in the center of NGC 6764, Kohno et al. (2001) classified this galaxy as a combined AGN/starburst, based on the high $\mathrm{HCN} / \mathrm{CO}$ line ratio $(\sim 0.7)$ in the nucleus which suggests the presence of nuclear activity. Recently Hota \& Saikia (2006, hereafter HS06) presented GMRT+VLA radio continuum and HI observations together with $\mathrm{H} \alpha$ data of the galaxy NGC 6764 where they found bipolar radio bubbles along the minor axis together with a compact core. Their HI observations reveal two peaks of emission at the ends of the bar and a depletion in the center together an HI absorption towards the radio core. The coexistence of an AGN and a starburst nucleus in NGC 6764 makes this galaxy an ideal target for studying the influence of both processes.

We present new high spatial resolution observations of the ${ }^{12} \mathrm{CO}(1-0)$ and ${ }^{12} \mathrm{CO}(2-1)$ lines made with the IRAM Plateau de Bure Interferometer $\left(\mathrm{PdBI}^{1}\right)$. We also present high resolution $\mathrm{VLA}^{2}$ radio continuum maps at $3.5 \mathrm{~cm}$ and $20 \mathrm{~cm}$ (Laine et al. 2006). We describe our observations in Sect. 2. In Sect. 3 we discuss the thermal and synchrotron emission associated with the hot ISM, and their connection to the nuclear star formation. The distribution, kinematics, physical properties, and mass of the molecular gas in the outflow in NGC 6764 are described in Sect. 4. The energies involved in the outflow as determined by the molecular gas and radio continuum observations of the outflow are addressed in Sect. 5. We discuss our results in Sect. 6 and summarize them in Sect. 7. Throughout this paper, we use the following cosmological parameters: $H_{0}=75 \mathrm{~km} \mathrm{~s}^{-1}$, $\Omega_{\Lambda}=0.7$ and $\Omega_{\mathrm{b}}=0.3$.

\section{Observations and data reduction}

\subsection{Radio continuum data}

The radio continuum data were obtained with the Very Large Array (VLA) at 3.5 and $20 \mathrm{~cm}$ in $\mathrm{C}$ and A configurations (see Fig. 1), respectively. We obtained a comparable spatial resolution at the two observed wavelengths, $1^{\prime \prime} .39 \times 1^{\prime \prime} .26$ $\left(\mathrm{PA}=-45^{\circ}\right)$ at $20 \mathrm{~cm}$ and $2^{\prime \prime} .50 \times 2^{\prime \prime}\left(\mathrm{PA}=15.5^{\circ}\right)$ at $3.5 \mathrm{~cm}$. For further details about the radio continuum observations, see Laine et al. (2006). The peak position of the $20 \mathrm{~cm}$ emission, $\alpha_{20 \mathrm{~cm}}(\mathrm{~J} 2000)=19: 08: 16.316, \delta_{20 \mathrm{~cm}}(\mathrm{~J} 2000)=50: 55: 59.37$, was determined to an accuracy better than $0 . ' 1$, by filtering out large spatial frequencies, using a wavelet transformation (e.g., Leon et al. 2000), and fitting a Gaussian to the remaining emission.

\subsection{CO data}

The ${ }^{12} \mathrm{CO}(1-0)$ and ${ }^{12} \mathrm{CO}(2-1)$ lines were observed with the IRAM interferometer (see Table 2), located on the Plateau de Bure (France), in the B and C configurations of the array, in 1998. We calibrated the visibilities using the radio galaxy 3C 380 as an amplitude calibrator and 1823+568 and 3C 380 as phase references. The Plateau de Bure Interferometer (PdBI) was phase-centered at $\alpha_{J 2000}=19: 08: 16.370$ and $\delta_{J 2000}=+50: 55: 59.58$. The velocity resolution was set at $10 \mathrm{~km} \mathrm{~s}^{-1}$ for the two CO transitions, and the CLEANed maps have been restored with a synthesized beam of $2^{\prime \prime} 0 \times 11^{\prime \prime} 6$ $\left[\mathrm{PA}=7^{\circ} ;{ }^{12} \mathrm{CO}(1-0)\right]$, and $1^{\prime \prime} .25 \times 1 . \prime 25\left[\mathrm{PA}=0^{\circ} ;{ }^{12} \mathrm{CO}(2-1)\right]$. The rms noise for the channel maps is $2 \mathrm{mJy}^{\text {beam }^{-1}}$ [58 mK; $\left.{ }^{12} \mathrm{CO}(1-0)\right]$, and $5.5 \mathrm{mJy} \mathrm{beam}^{-1}$ [82.5 $\mathrm{mK}$; $\left.{ }^{12} \mathrm{CO}(2-1)\right]$. Hereafter the velocity reference is fixed to $2400 \mathrm{~km} \mathrm{~s}^{-1}$, giving a redshifted frequency of $114.3489 \mathrm{GHz}$ for the ${ }^{12} \mathrm{CO}(1-0)$ observations, and $228.6932 \mathrm{GHz}$ for the ${ }^{12} \mathrm{CO}(2-1)$ observations.

The total integrated ${ }^{12} \mathrm{CO}(1-0)$ flux is $225 \mathrm{Jy} \mathrm{km} \mathrm{s}^{-1}$ in the $55^{\prime \prime}$ beam of the NRAO 12-m telescope (Sanders \& Mirabel 1985). Our PdBI ${ }^{12} \mathrm{CO}(1-0)$ observations detected a total flux of $112 \mathrm{Jy} \mathrm{km} \mathrm{s}^{-1}$, i.e., filtering out about $50 \%$ of the total flux, assuming all the emission is concentrated in the $45^{\prime \prime}$ primary beam of the PdBI. This implies that a large fraction of the molecular gas must be in an extended smooth component to which the interferometer is insensitive. From Eckart et al. (1991) we estimate with IRAM-30 m observations the ${ }^{12} \mathrm{CO}(2-1)$ integrated

\footnotetext{
1 Based on observations carried out with the IRAM Plateau de Bure Interferometer. IRAM is operated by CNRS (France), the MPG (Germany) and the IGN (Spain).

2 The VLA is a research facility of the National Radio Astronomy Observatory (NRAO), which is operated by Associated Universities Inc., under contract with the National Science Foundation.
} 

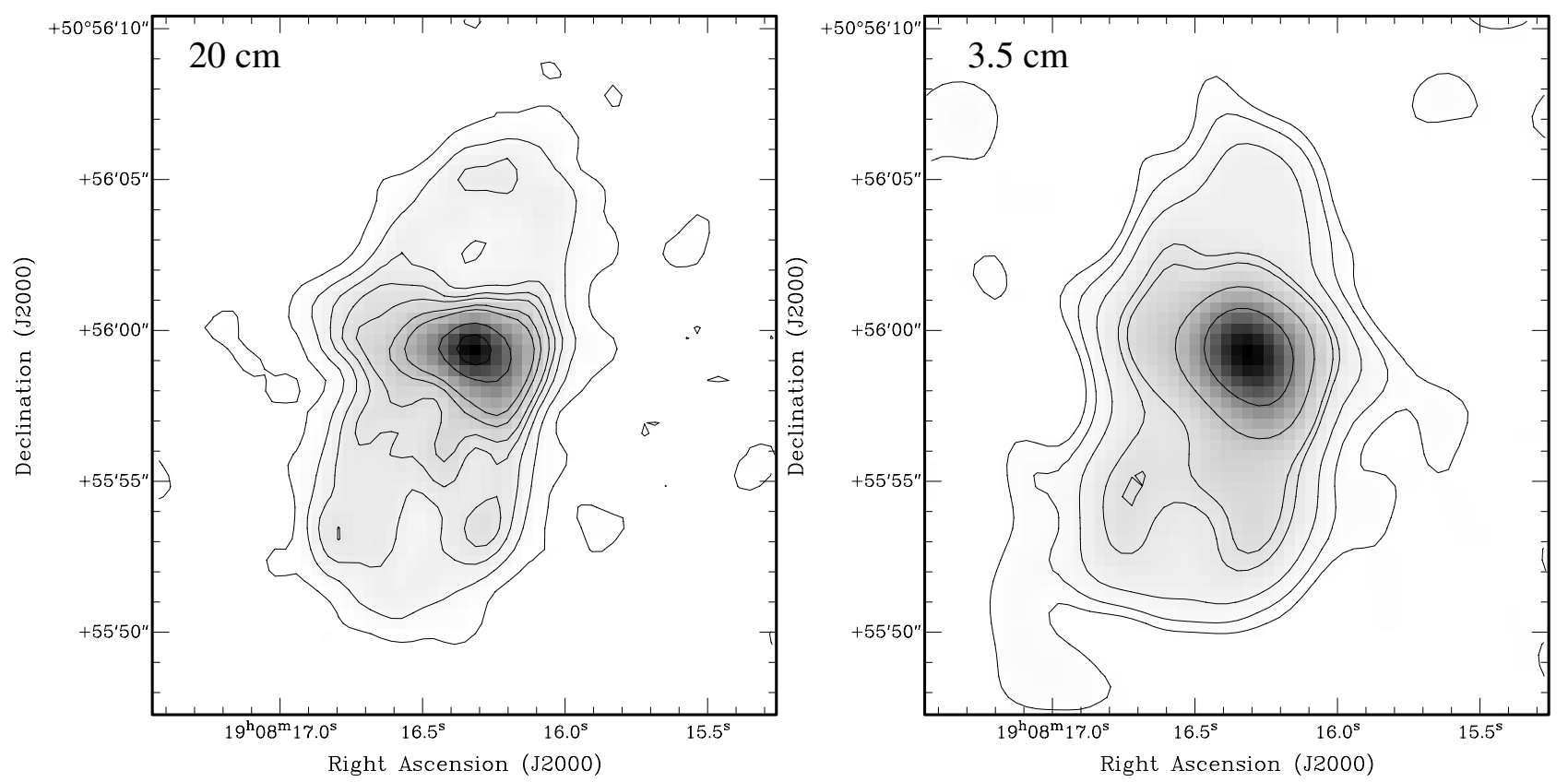

Fig. 1. Left: radio continuum emission at $20 \mathrm{~cm}$ with a synthesized beam of 1 '” $39 \times 1$.'26. The contour levels are at $(0.1,0.5,1,1.5,2,3,4,8$, 12) $\mathrm{mJy} \mathrm{beam}^{-1}$ and the rms noise is $70 \mu \mathrm{Jy}_{\text {beam }}{ }^{-1}$. Right: radio continuum emission at $3.5 \mathrm{~cm}$ (VLA) with a synthesized beam of 2 '. $50 \times 22^{\prime \prime} 0$. The contour levels are at $(0.1,0.2,0.4,0.8,1,2,4,8) \mathrm{mJy} \mathrm{beam}^{-1}$ and the rms noise is $56 \mu \mathrm{Jy}_{\text {beam }}{ }^{-1}$.
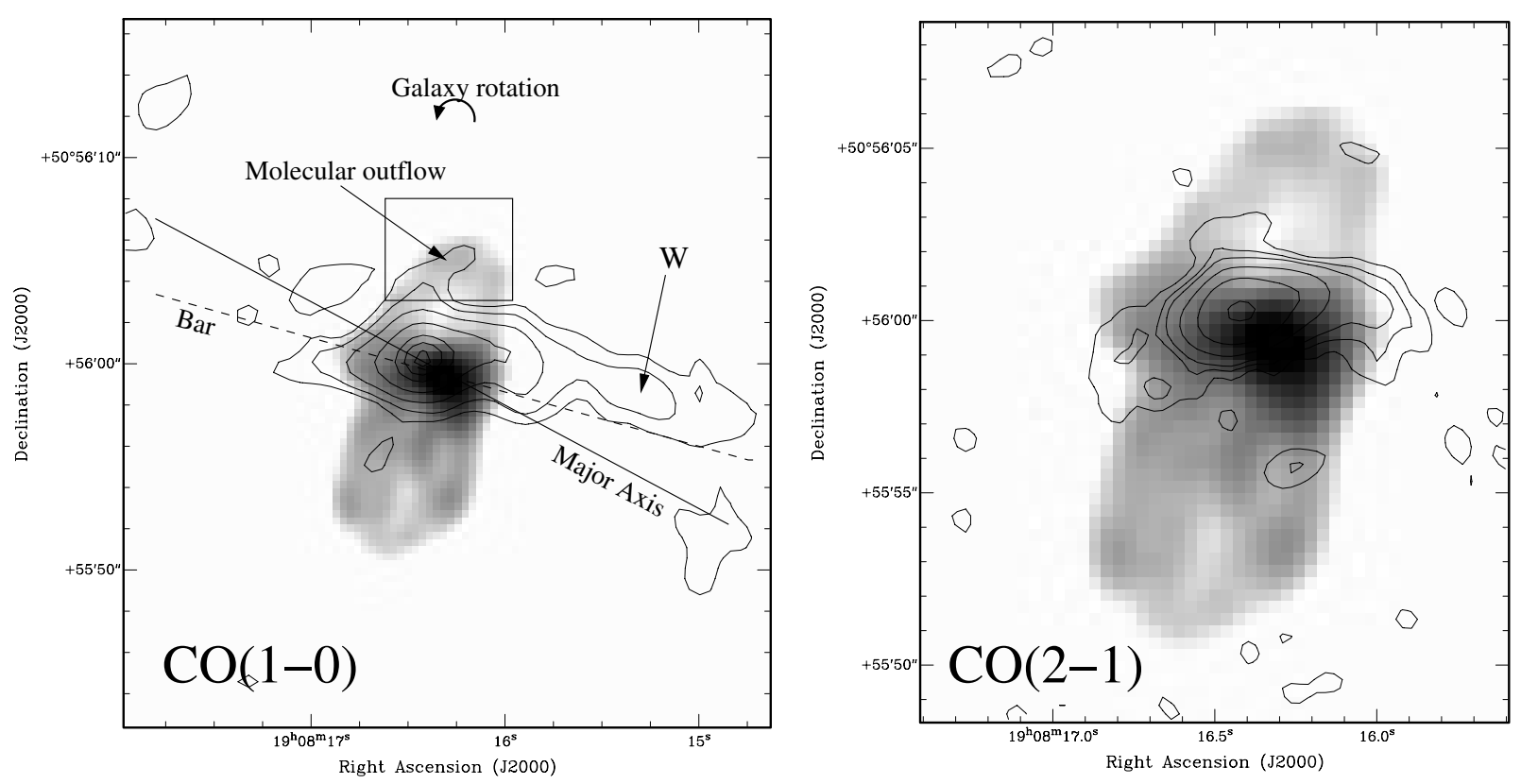

Fig. 2. Left: VLA $20 \mathrm{~cm}$ radio continuum emission (greyscale) overlaid on the integrated intensity image of the ${ }^{12} \mathrm{CO}(1-0)$ emission. Right: ${ }^{12} \mathrm{CO}(2-1)$ integrated intensity image. The contours for ${ }^{12} \mathrm{CO}(1-0)$ are at $(1,2,4,8,15,20,25) \mathrm{Jy} \mathrm{beam}^{-1} \mathrm{~km} \mathrm{~s}^{-1}$, and for ${ }^{12} \mathrm{CO}(2-1)$ at $(2,4,6$, $12,24,52$,) Jy beam ${ }^{-1} \mathrm{~km} \mathrm{~s}^{-1}$. The position of the major axis of the galaxy and of the bar are shown by solid and dashed lines, respectively.

intensity to be $\sim 480 \mathrm{Jy} \mathrm{km} \mathrm{s}^{-1}$ in the center of NGC 6764 , which implies a filtering of about $65 \%$ of the ${ }^{12} \mathrm{CO}(2-1)$ flux by the PdBI observations.

The integrated intensity maps for the ${ }^{12} \mathrm{CO}(1-0)$ and ${ }^{12} \mathrm{CO}(2-1)$ emission lines are shown in Fig. 2. The position of the $\mathrm{CO}$ emission peak $\alpha_{\mathrm{CO}}(\mathrm{J} 2000)=19: 08: 16.402$, $\delta_{\mathrm{CO}}(\mathrm{J} 2000)=50: 56: 0.29$ has been estimated using the higher spatial resolution of the ${ }^{12} \mathrm{CO}(2-1)$ transition. Assuming that the spiral arms are trailing, and noting that the receding velocities in the disk are on the eastern side of NGC 6764, the northern side of the galaxy disk is closer to us.

\section{Thermal and synchrotron emission}

\subsection{Radio continuum}

The total integrated $20 \mathrm{~cm}$ flux density from the VLA observations is $90 \mathrm{mJy}$, which compared to the single dish flux density of $133 \mathrm{mJy}$ (White \& Becker 1992), implies that $70 \%$ of the total flux density is recovered by the VLA observations. The total $3.5 \mathrm{~cm}$ flux density is $31 \mathrm{mJy}$. Both VLA maps at 20 and $3.5 \mathrm{~cm}$ present the same features with a better spatial resolution at $20 \mathrm{~cm}$ (see Fig. 1). A bright peak is observed towards the nucleus of NGC 6764. Two 5" spurs emerge from the unresolved 


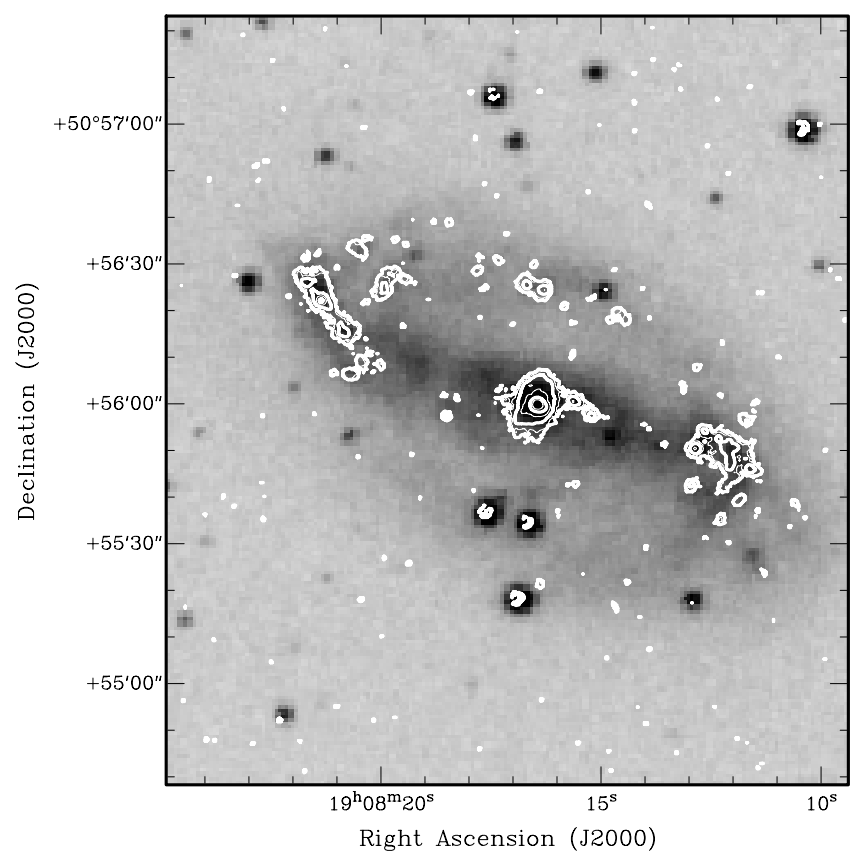

Fig. 3. B image (from Digital Sky Survey) of NGC 6764 overlaid by $\mathrm{H} \alpha$ emission contours. The contours are spaced by 0.5 dex steps, starting at the $0.5 \sigma$ level.

nucleus, towards the south and the south-east. The emission near the nucleus is not consistent with a just a nuclear point source but hints at the presence of a nuclear radio jet, as proposed by HS06. The most remarkable feature is the shell-like emission, at both wavelengths, centered on the central peak with its major axis perpendicular to the major axis of NGC 6764 and extending about $1 \mathrm{kpc}$ above and below the disk plane of the highly inclined galaxy NGC 6764. The clumpy shell emission is brighter towards south than towards north. These radio bubbles were previously reported by Baum et al. (1993), HS06 and Laine et al. (2006).

\section{2. $\mathrm{H} \alpha$ emission}

An H $\alpha$ image of NGC 6764 was kindly given to us by D. Frayer. The observations were done at the Palomar 60-inch telescope with a seeing of $1-3^{\prime \prime}$, using the X-filter centered at $6616 \AA$ with a width of $20 \AA$, leaving out most of the [NII] emission line. Figure 3 shows the $\mathrm{H} \alpha$ emission at the center of NGC 6764. Apart from the most intense $\mathrm{H} \alpha$ emission in the central region, two other regions of emission are seen near the tip of the bar, which also lies near the beginning of the spiral arms. The most striking characteristic of the $\mathrm{H} \alpha$ emission near the center of NGC 6764 is its bubble-like shape, but its size is smaller than the size of the radio continuum outflow feature in the south. In Fig. 4 a $4^{\prime \prime}$ spur is observed to the southeast of the location of the ${ }^{12} \mathrm{CO}(2-1)$ peak, bending towards south. This spur is spatially correlated with faint $\left(<6 \mathrm{Jy} \mathrm{beam}^{-1} \mathrm{~km} \mathrm{~s}^{-1}\right)$ ${ }^{12} \mathrm{CO}(2-1)$ emission.

In Fig. 5 the $\mathrm{H} \alpha$ emission is compared with the radio continuum spectral index. The "hot spot" in the bubble cavity with a flat spectral index $(\alpha \sim-0.2)$ is associated with $\mathrm{H} \alpha$ emission driven by the nuclear starburst. Similarly, the tip of the southeastern $\mathrm{H} \alpha$ emission spur coincides with a small "hot spot" that has a flat spectrum.

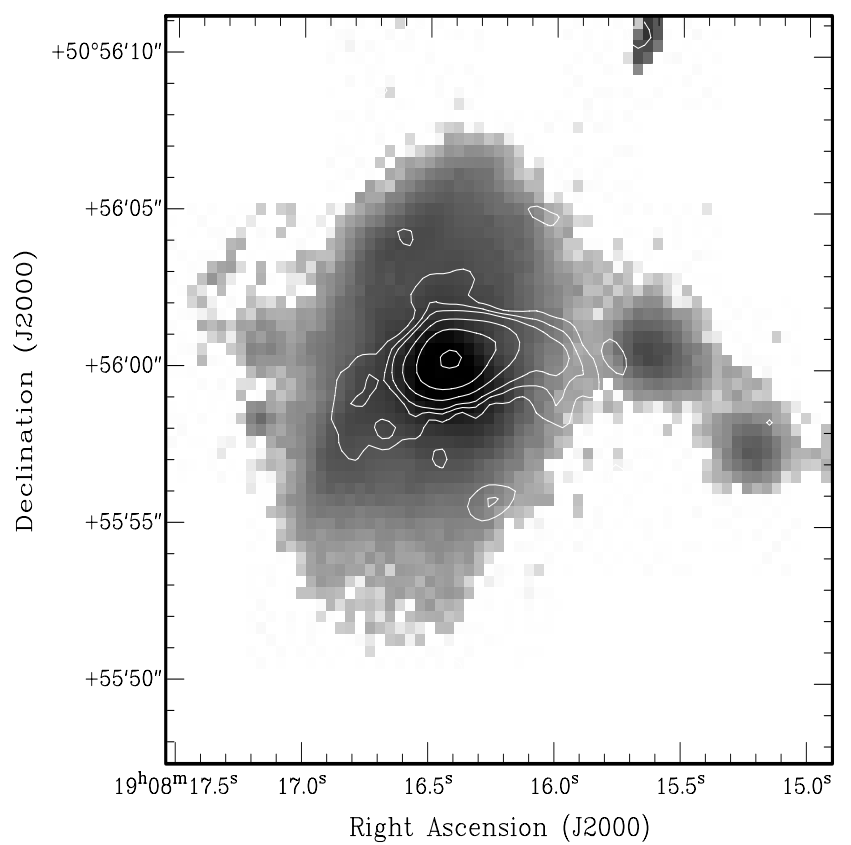

Fig. 4. $\mathrm{H} \alpha$ emission (greyscale) overlaid by the ${ }^{12} \mathrm{CO}(2-1)$ emission contours (same levels as in Fig. 2).

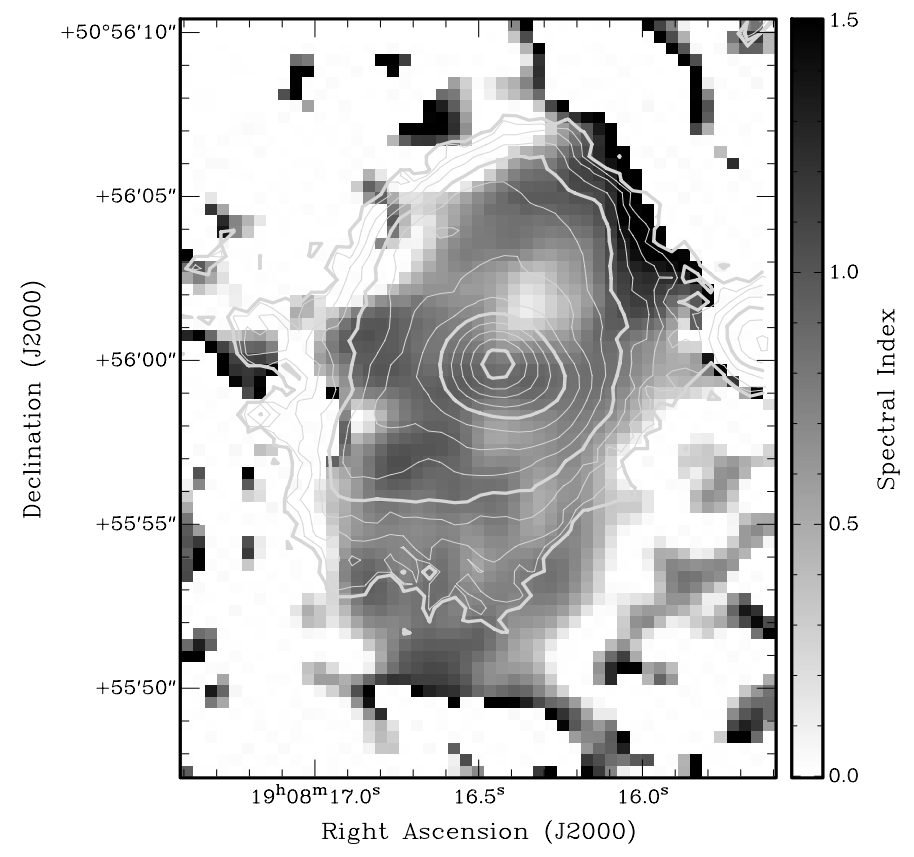

Fig. 5. Spectral index $-\alpha$ between $3.5 \mathrm{~cm}$ and $20 \mathrm{~cm}$ (greyscale), overlaid by $\mathrm{H} \alpha$ emission contours.

\subsection{Nuclear star formation}

The nuclear star formation history was studied in detail by Schinnerer et al. (2000) using near-infrared K-band imaging spectroscopy of the very center of NGC 6764. To get the current star formation rate we have used here the FIR and the $\mathrm{H} \alpha$ emission line. We estimate as well the contribution of the thermal and non-thermal radiocontiuum emission. 
Table 1. Global properties of NGC 6764.

\begin{tabular}{lll}
\hline \hline Property & Value & References \\
\hline Hubble type & SB(s)bc & NED \\
Distance (Mpc) & 32 & $H_{0}=75 \mathrm{~km} \mathrm{~s}^{-1} \mathrm{Mpc}^{-1}$ \\
Inclination & $61.5^{\circ}$ & LEDA \\
Major Axis PA & $62^{\circ}$ & LEDA \\
Bar PA & $74.6^{\circ}$ & LEDA \\
$S_{12} \mu \mathrm{m}(\mathrm{Jy})$ & 0.36 & NED \\
$S_{25 \mu \mathrm{m}(\mathrm{Jy})}$ & 1.29 & NED \\
$S_{60} \mu \mathrm{m}(\mathrm{Jy})$ & 6.33 & NED \\
$S_{1000} \mu \mathrm{m}(\mathrm{Jy})$ & 11.56 & NED \\
$L_{\mathrm{FIR}}\left(10^{10} L_{\odot}\right)$ & 1.1 & \\
$L_{\mathrm{H}} \alpha\left(10^{40} \mathrm{erg} \mathrm{s} \mathrm{s}^{-1}\right)$ & 23 & \\
$M_{\mathrm{HI}}\left(10^{9} \mathcal{M}_{\odot}\right)$ & 7.0 & NED \\
$S_{90 \mathrm{~cm}}(\mathrm{mJy})$ & 309 & WENSS \\
$S_{80 \mathrm{~cm}}(\mathrm{mJy})$ & 244 & Douglas et al. $(1996)$ \\
$S_{20 \mathrm{~cm}}(\mathrm{mJy})$ & 133 & White \& Becker $(1992)$ \\
$S_{6 \mathrm{~cm}}(\mathrm{mJy})$ & 34 & Becker et al. (1991) \\
$S_{3.5 \mathrm{~cm}}(\mathrm{mJy})$ & 31 & This paper \\
\hline
\end{tabular}

Table 2. PdBI observations.

\begin{tabular}{lll}
\hline \hline Parameter & ${ }^{12} \mathrm{CO}(1-0)$ & ${ }^{12} \mathrm{CO}(2-1)$ \\
\hline Field of view & $45^{\prime \prime}(6.9 \mathrm{kpc})$ & $22^{\prime \prime}(3.4 \mathrm{kpc})$ \\
Bandwidth & $270 \mathrm{MHz}$ & $473 \mathrm{MHz}$ \\
Velocity resolution & $10 \mathrm{~km} \mathrm{~s}^{-1}$ & $10 \mathrm{~km} \mathrm{~s}^{-1}$ \\
Synthesized beam size & $2^{\prime \prime} \times 1^{\prime \prime} 6, \mathrm{PA} 7^{\circ}$ & $1^{\prime \prime} .25 \times 1^{\prime \prime} 25$ \\
rms noise (per channel) & $2 \mathrm{mJy} \mathrm{beam}^{-1}$ & $6 \mathrm{mJy} \mathrm{beam}^{-1}$ \\
\hline
\end{tabular}

\subsubsection{Star formation rate}

The total FIR luminosity is computed for the whole galaxy from the 60 and $100 \mu \mathrm{m}$ IRAS fluxes (see Table 1), but is very close to the bolometric value $L_{\mathrm{bol}}=9 \times 10^{9} L_{\odot}$ computed for the nuclear region by Schinnerer et al. (2000). Following Condon (1992), the star formation rate $(\mathrm{SFR})$ for massive stars is $\operatorname{SFR}\left(M>5 \mathcal{M}_{\odot}\right)=$ $9.1 \times 10^{-11}\left(L_{\mathrm{FIR}} / L_{\odot}\right)$, which gives a SFR of $1 \mathcal{M}_{\odot} / \mathrm{yr}$.

The $\mathrm{H} \alpha$ line is mainly emitted by HII regions where newborn massive stars are ionizing the gas. The total $\mathrm{H} \alpha$ flux can be used as a tracer of the star formation rate (Kennicutt 1998):

$$
L(\mathrm{H} \alpha)(\mathrm{erg} / \mathrm{s})=1.26 \times 10^{41} \operatorname{SFR}\left(\mathcal{M}_{\odot} / \mathrm{yr}\right) .
$$

The H $\alpha$ flux is $1.3 \times 10^{41} \mathrm{erg} / \mathrm{s}$ for the nuclear starburst for an aperture size of $3^{\prime \prime}$ (see Table 3 ), which gives a SFR of $1 \mathcal{M}_{\odot} / \mathrm{yr}$, consistent with the SFR derived from the FIR luminosity. These two derivations of the SFR take into account only massive stars. Including all stellar masses, and accounting for the effects of optical extinction, Eckart et al. (1991) derived a SFR of $4 \mathcal{M}_{\odot} / \mathrm{yr}$ for the nuclear starburst (NSB), and a supernova rate of $1.2 \times$ $10^{-2} / \mathrm{yr}$.

\subsection{2. (Non-)thermal radio continuum}

While the radio continuum emission at long wavelengths is mainly produced by the compact source (AGN) the contribution from the NSB is important at $3.5 \mathrm{~cm}$ creating a flatter spectrum. By fitting the radio continuum between $6 \mathrm{~cm}$ and $80 \mathrm{~cm}$ where the non-thermal contribution is dominant, we found a spectral index of $\alpha=-0.46$. Extrapolating that fit down to $3.5 \mathrm{~cm}$, we obtained a non-thermal flux density of $6 \mathrm{mJy}$, which is much below the measured value of $31 \mathrm{mJy}$.

The free-free contribution ("bremsstrahlung") can be computed from the $\mathrm{H} \alpha$ flux. The Balmer lines provide an estimate of
Table 3. Outflow bubble and nuclear contributions to the $\mathrm{H} \alpha$ and $20 \mathrm{~cm}$ radio continuum emission derived by fitting a 2D Gaussian to the central emission. The molecular gas mass for the NSB is the mass encompassed by one ${ }^{12} \mathrm{CO}(1-0)$ beam, and has a projected size of $310 \times 250 \mathrm{pc}$.

\begin{tabular}{lllll}
\hline \hline & $\begin{array}{l}\mathrm{H} \alpha \\
\left(10^{39} \mathrm{erg} \mathrm{s}^{-1}\right)\end{array}$ & $\begin{array}{l}20 \mathrm{~cm} \\
(\mathrm{mJy})\end{array}$ & $\begin{array}{l}3.5 \mathrm{~cm} \\
(\mathrm{mJy})\end{array}$ & $\begin{array}{l}M\left(\mathrm{H}_{2}\right) \\
\left(\mathcal{M}_{\odot}\right)\end{array}$ \\
\hline Nucleus & $\sim 130$ & 90 & 28 & $4.3 \times 10^{8}$ \\
Outflow & $\sim 45$ & 45 & 12 & $1.7 \times 10^{7}$ \\
\hline
\end{tabular}

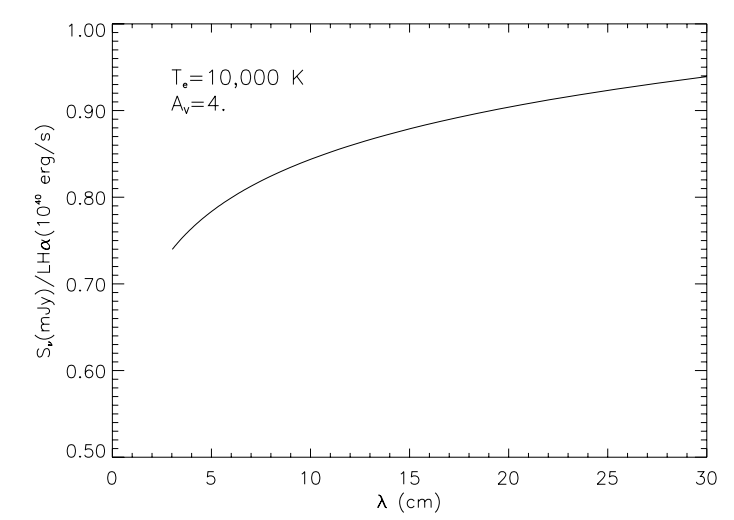

Fig. 6. Ratio of the thermal radio continuum emission (mJy) and $\mathrm{H} \alpha \mathrm{lu}-$ minosity $\left(10^{40} \mathrm{erg} / \mathrm{s}\right)$ at the distance of NGC 6764 , assuming an electron temperature of $10000 \mathrm{~K}$ and an optical extinction of $A_{\mathrm{V}}=4$.

Table 4. Thermal (free-free) and non-thermal (synchrotron) contribution to the observed radio continuum emission at different wavelengths at the center of NGC 6764. The thermal contribution is estimated from the $\mathrm{H} \alpha$ luminosity (see text).

\begin{tabular}{llll}
\hline \hline Wavelength & $\begin{array}{l}\text { Thermal } \\
(\mathrm{mJy})\end{array}$ & $\begin{array}{l}\text { Non-thermal } \\
(\mathrm{mJy})\end{array}$ & Ratio $\frac{\text { Thermal }}{\text { Total }}$ \\
\hline $20 \mathrm{~cm}$ & 17.6 & 115.4 & $13 \%$ \\
$6 \mathrm{~cm}$ & 15.6 & 18.4 & $46 \%$ \\
$3.5 \mathrm{~cm}$ & 14.8 & 16.3 & $48 \%$ \\
\hline
\end{tabular}

the number of ionizing photons present in the source. If the optical extinction is known, the $\mathrm{H} \alpha$ flux can be used to compute the thermal radio continuum flux (e.g., Caplan \& Deharveng 1986). Assuming an electron temperature of $10^{4} \mathrm{~K}$ and an optical extinction of $A_{\mathrm{V}}=4$ (Eckart et al. 1991), the thermal radio continuum flux density at $3.5 \mathrm{~cm}$ is $14.8 \mathrm{mJy}$, obtained by integrating the $\mathrm{H} \alpha$ luminosity in the outflow. The $\mathrm{H} \alpha$ extinction is given by $A_{\mathrm{H} \alpha}=0.605 * A_{\mathrm{V}}$. Figure 6 shows the ratio between the radio flux density (mJy) and the $\mathrm{H} \alpha$ luminosity (in units of $10^{40} \mathrm{erg} / \mathrm{s}$ ) for the thermal (free-free) contribution versus wavelength. This ratio is $75 \%$ at $3.5 \mathrm{~cm}$. The maximum thermal contribution to the flux density at $3.5 \mathrm{~cm}$ is thus about $23 \mathrm{mJy}$ (Zurita et al. 2000) which is consistent with our previous estimation of $6 \mathrm{mJy}$ for the non-thermal radio continuum.

Table 4 gives an estimate of the thermal and non-thermal contributions to the observed radio continuum emission, using the $\mathrm{H} \alpha$ luminosities to estimate the thermal component. At $20 \mathrm{~cm}$ the radio continuum emission is mainly non-thermal $(87 \%)$, but at shorter wavelengths $(6$ and $3.5 \mathrm{~cm})$ both contributions are comparable. 


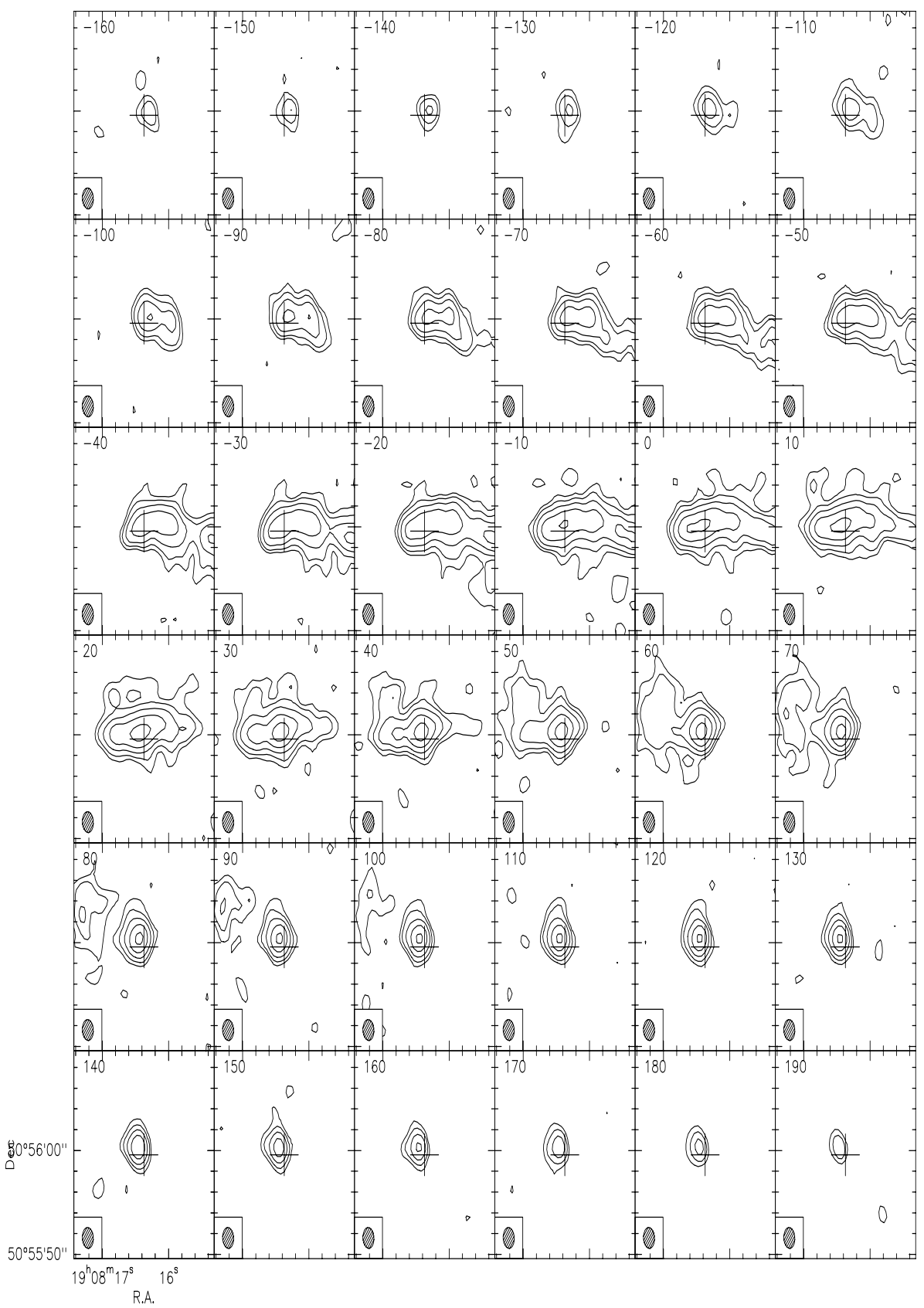

Fig. 7. Channel maps for the ${ }^{12} \mathrm{CO}(1-0)$ emission. The rms noise is 2 mJy beam ${ }^{-1}$. The contours are at $(3,6$, $12,24,48,92) \sigma$. The synthesized beam is $2^{\prime \prime} \times 1^{\prime \prime} 6$ in size. The rest velocity is 2400 $\mathrm{km} \mathrm{s}^{-1}$. The cross is located at the phase center, i.e., at $\alpha_{J 2000}=19: 08: 16.370$ and $\delta_{J 2000}=50: 55: 59.58$.

\section{Molecular gas}

\subsection{Distribution}

The CO emission detected with the PdBI is strongly concentrated toward the center of NGC 6764 (see Fig. 2) and reaches a maximum at a projected distance of 1 '.25 ( 190 pc) from the radio continuum peak. More than $98 \%$ of the total molecular gas is concentrated within the inner $800 \mathrm{pc}$, and as much as $20 \%$ of the total ${ }^{12} \mathrm{CO}(1-0)$ emission is included within one beam.

Apart from the central concentration, the $\mathrm{CO}$ emission is extended along the bar towards the western side ("W") and has a smaller extension to the eastern side. Assuming that the external spiral arms are trailing, this $\mathrm{CO}$ emission is on the leading side of the bar.

The western $\mathrm{CO}$ feature, which extends to $10^{\prime \prime}$ from the center, is aligned with the primary bar. The $\mathrm{CO}$ emission is associated with two $\mathrm{H} \alpha$-emitting star forming regions (with $5 \times 10^{39}$ and $3 \times 10^{39} \mathrm{erg} \mathrm{s}^{-1} \mathrm{H} \alpha$ luminosities not corrected for extinction) which are among the brightest in NGC 6764, excluding the strong $\mathrm{H} \alpha$ emission at the tip of the bar (Rozas et al. 1996). Since NGC 6764 is highly inclined, the location of star formation and molecular gas in these regions is not completely clear.

Figure 7 shows the ${ }^{12} \mathrm{CO}(1-0)$ channel maps at $10 \mathrm{~km} \mathrm{~s}^{-1}$ velocity resolution relative to systemic. ${ }^{12} \mathrm{CO}(1-0)$ emission is detected in the individual channels at $2.5 \sigma$ level from 2210 to $2600 \mathrm{~km} \mathrm{~s}^{-1}$ (LSR). Two ${ }^{12} \mathrm{CO}(1-0)$ peaks are seen in the very center at negative and positive velocities. The first of these strong peaks is seen mainly at negative velocities $\left(-110\right.$ to $\left.-20 \mathrm{~km} \mathrm{~s}^{-1}\right)$, and is separated from the primary $\mathrm{CO}$ peak by $\sim 2^{\prime \prime}$. This secondary peak is not visible in the integrated ${ }^{12} \mathrm{CO}(1-0)$ map. Extended weaker features are visible in the channel maps as well. One feature is seen north of the ${ }^{12} \mathrm{CO}(1-0)$ peak at velocities -40 to $130 \mathrm{~km} \mathrm{~s}^{-1}$. Another more extended feature (see Fig. 8) is seen at velocities 20 to $100 \mathrm{~km} \mathrm{~s}^{-1}$. The first feature is associated with the molecular outflow, visible in the ${ }^{12} \mathrm{CO}(1-0)$ integrated intensity image, and flaring up to a 


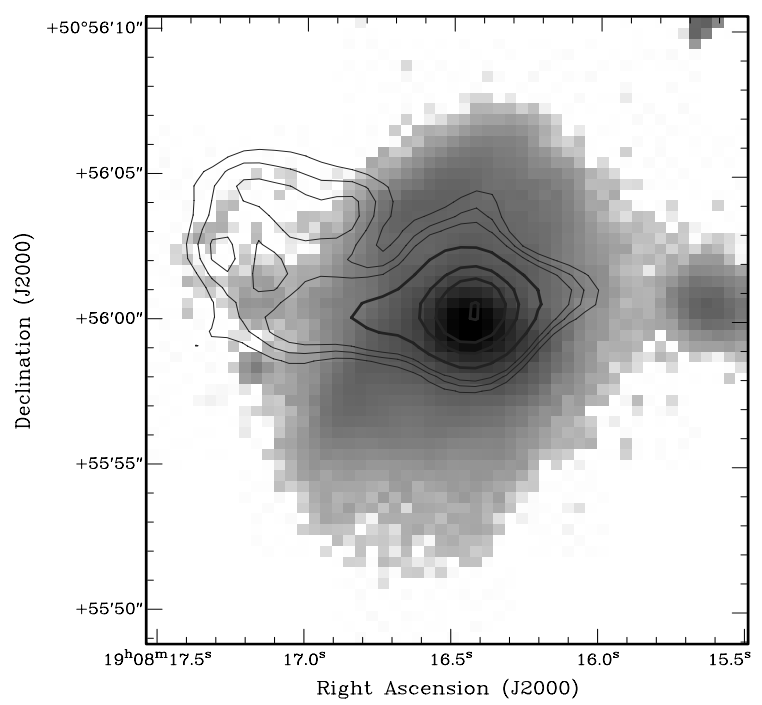

Fig. 8. Integrated ${ }^{12} \mathrm{CO}(1-0)\left(\mathrm{Jy} \mathrm{beam}^{-1} \mathrm{~km} \mathrm{~s}^{-1}\right)$ emission intensity in the velocity range $20-100 \mathrm{~km} \mathrm{~s}^{-1}$ overlaid on the $\mathrm{H} \alpha$ emission (greyscale). The contour levels are 0.5, 0.75, 1, 2, 4, 6 and $10 \mathrm{Jy}_{\text {beam }}{ }^{-1} \mathrm{~km} \mathrm{~s}^{-1}\left(1 \sigma \mathrm{rms}\right.$ noise $\left.\sim 0.02 \mathrm{Jy}_{\text {beam }}{ }^{-1} \mathrm{~km} \mathrm{~s}^{-1}\right)$.

$8^{\prime \prime}(\sim 1 \mathrm{kpc})$ height. Correcting for the disk inclination, the molecular gas reaches $1.5 \mathrm{kpc}$ above the plane of the galaxy. The velocities are consistent with motion perpendicular to the galaxy plane, taking into account the inclination and orientation of NGC 6764.

The faint extended feature between 20 and $100 \mathrm{~km} \mathrm{~s}^{-1}$ covers an area of about $1 \mathrm{kpc}^{2}$ between $5^{\prime \prime}$ and $10^{\prime \prime}$ radii (800-1600 pc). At a velocity of $70 \mathrm{~km} \mathrm{~s}^{-1}$, it reaches a total length of $12^{\prime \prime}(\sim 1.9 \mathrm{kpc})$, and is oriented north to south. The velocity field shows few signs of outflow motion in this region, indicating that this feature is in the plane of the galaxy. The western feature (W) has a large velocity extent from $-80 \mathrm{~km} \mathrm{~s}^{-1}$ up to $10 \mathrm{~km} \mathrm{~s}^{-1}$. A CO spur is visible towards the north, and its location is spatially correlated with the boundary of the radio continuum superbubble, as seen at $20 \mathrm{~cm}$.

\subsection{Kinematics}

\subsubsection{First and second velocity moments}

Figure 9 shows the beam-smeared map of the intensity-weighted ${ }^{12} \mathrm{CO}(2-1)$ velocity field, derived from the first moment of the ${ }^{12} \mathrm{CO}(2-1)$ data cube, overlaid on the $20 \mathrm{~cm}$ radio continuum emission. We determined the dynamical center of the gas motion from the ${ }^{12} \mathrm{CO}(2-1)$ data, and compared it to the positions of the various emission peaks (see Table 5). HS06 find an offset of $0.55^{\prime \prime}$ between the VLA A-array peak at $3.5 \mathrm{~cm}$ and the optical nucleus. They explain that difference by a higher extinction close to the nucleus, which is consistent with the molecular gas distribution. The dynamical center of $\mathrm{CO}$ emission is spatially coincident with the nucleus as seen in the $B$ - and $I$-bands, and located about 0.5 ( $~ 80$ parsecs) from the radio continuum peak. Our observations are consistent with the global rotation of the galaxy disk, combined with streaming motions along the bar and/or a warping of the disk. The strongest deviation from the rotation pattern, the so-called "spider diagram", is seen on the eastern side along the molecular outflow, which originates from the ${ }^{12} \mathrm{CO}(1-0)$ peak, at $1^{\prime \prime}-2^{\prime \prime}$ to the NE of the radio continuum peak. The noise in the velocity field there is significant since the $\mathrm{CO}$ emission is centrally concentrated and the emission intensity is much lower beyond the peak. The position angle (PA) of the

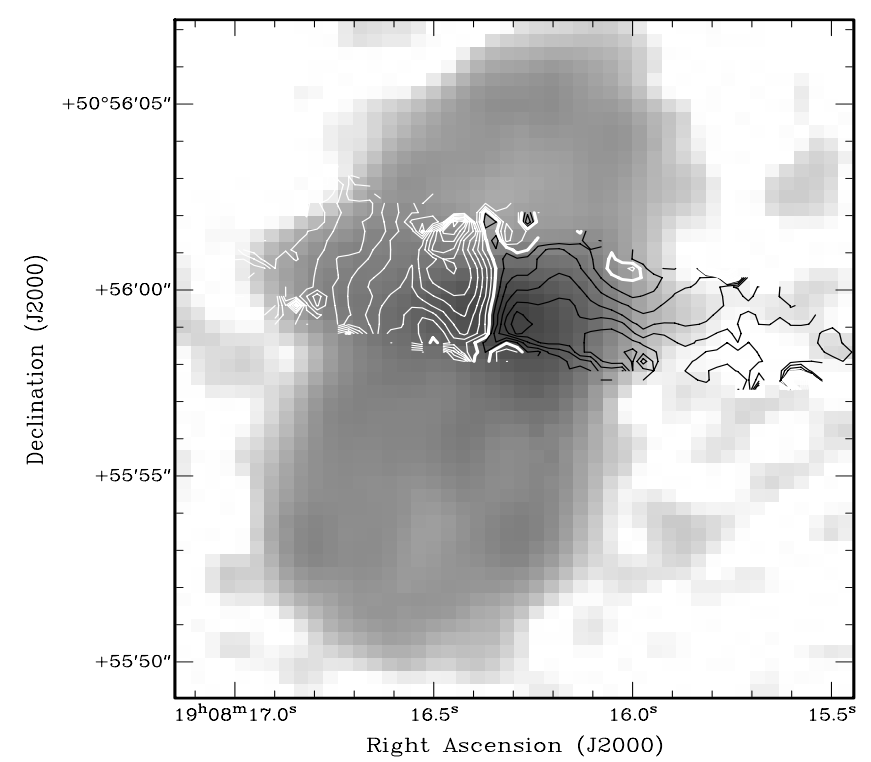

Fig. 9. Isovelocity contours of the ${ }^{12} \mathrm{CO}(2-1)$ emission overlaid on a color image of the $20 \mathrm{~cm}$ radio continuum emission. Black contour lines indicate negative velocities, and are separated by steps of $10 \mathrm{~km} \mathrm{~s}^{-1}$. White contour lines imply positive velocities with respect to the reference velocity, and are also separated by steps of $10 \mathrm{~km} \mathrm{~s}^{-1}$. The reference velocity is $2400 \mathrm{~km} \mathrm{~s}^{-1}$.

galaxy major axis, determined from the velocity field, $70^{\circ} \pm 5$, is slightly larger than the PA from the orientation of the optical isophotes (61.5). The HI data of Wilcots et al. (2001) indicate a change in the PA between the outer part $\left(\sim 58^{\circ}\right)$ and the inner part of the disk $\left(\sim 76^{\circ}\right)$. The difference is most likely due to the streaming motion near the center caused by the bar, as confirmed by the disturbed $\mathrm{CO}$ velocity field.

Figure 10 shows the beam-smeared map of the intensityweighted ${ }^{12} \mathrm{CO}(1-0)$ velocity dispersion in the center, derived from the second moment of the ${ }^{12} \mathrm{CO}(1-0)$ data cube, overlaid with the ${ }^{12} \mathrm{CO}(1-0)$ velocity field. The central velocity dispersion $\sigma_{\mathrm{V}}=200 \mathrm{~km} \mathrm{~s}^{-1}$, is much higher than the internal velocity dispersion of a molecular cloud (typically a few $\mathrm{km} \mathrm{s}^{-1}$; see e.g. Scoville et al. 1987). More likely, it results from the beamsmeared velocity gradient caused by a steeply rising rotation curve, together with a contribution from streaming motions.

Within the astrometric accuracy the peak of the velocity dispersion is located at the position of the dynamical center. At the position of the molecular outflow the velocity dispersion has values up to $150 \mathrm{~km} \mathrm{~s}^{-1}$, whereas along the bar, at radii larger than $5^{\prime \prime}$ from the nucleus, the molecular gas velocity dispersion does not exceed $50 \mathrm{~km} \mathrm{~s}^{-1}$. Curiously, the velocity dispersion is asymmetric in the east-west direction, with larger values on the eastern side.

\subsubsection{Lindblad resonances}

The rotation curve has been computed from our ${ }^{12} \mathrm{CO}(1-0)$ data and the HI data from Wilcots et al. (2001), using the task INSPECTOR in Gipsy. Although the HI spatial resolution is poorer than in $\mathrm{CO}$, it provides data points at large radii. The rotation curve becomes flat at a value of $250 \mathrm{~km} \mathrm{~s}^{-1}$ at $\sim 12 \mathrm{kpc}$ from the center. Under the assumption of the epicyclic approximation, the azimuthal frequency $(\Omega)$ and the radial frequency $(\kappa)$ have been computed from the rotation curve. The bar pattern speed is estimated from the rotation curve and the length of the bar 
Table 5. Position (J2000.0) of the peak emission in radio continuum $(20 \mathrm{~cm}), \mathrm{CO}$, and $\mathrm{H} \alpha$ (off-filter and the emission line). The distance to the radio continuum peak is given in arcseconds.

\begin{tabular}{lllll}
\hline \hline Tracer & $\alpha_{2000}$ & $\delta_{2000}$ & $\begin{array}{l}D_{20} \\
\left({ }^{\prime \prime}\right)\end{array}$ & $\begin{array}{l}\text { Astrom. } \\
\text { accuracy } \\
\left({ }^{\prime \prime}\right)\end{array}$ \\
\hline $20 \mathrm{~cm}$ & $19: 08: 16.316$ & $50: 55: 59.37$ & & 0.1 \\
${ }^{12} \mathrm{CO}(1-0)_{\text {peak }}$ & $19: 08: 16.392$ & $50: 56: 0.24$ & 1.2 & 0.3 \\
${ }^{12} \mathrm{CO}(1-0)_{\text {vel.disp }}$ & $19: 08: 16.392$ & $50: 55: 59.88$ & 0.9 & 0.3 \\
${ }^{12} \mathrm{CO}(2-1)_{\text {dyn }}$ & $19: 08: 16.351$ & $50: 55: 59.74$ & 0.5 & 0.2 \\
${ }^{12} \mathrm{CO}(2-1)_{\text {peak }}$ & $19: 08: 16.402$ & $50: 56: 0.29$ & 1.2 & 0.2 \\
$\mathrm{H} \alpha$ & $19: 08: 16.440$ & $50: 55: 59.87$ & 1.3 & 0.2 \\
$\mathrm{H} \alpha_{\text {off }}$ & $19: 08: 16.362$ & $50: 55: 59.68$ & 0.5 & 0.2 \\
Nucleus $B$-band & $19: 08: 16.379$ & $50: 55: 59.82$ & 0.6 & 0.3 \\
Nucleus $I$-band & $19: 08: 16.374$ & $50: 55: 59.69$ & 0.6 & 0.3 \\
\hline
\end{tabular}

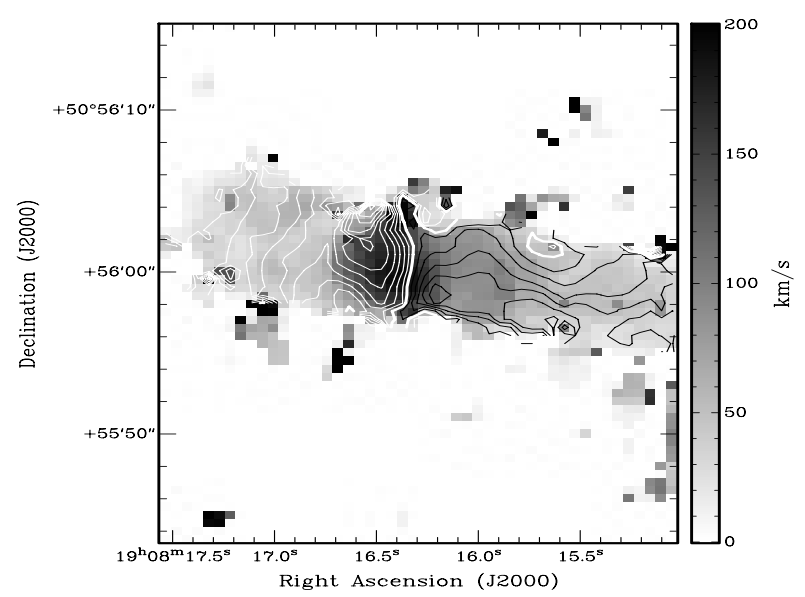

Fig. 10. Velocity dispersion of the ${ }^{12} \mathrm{CO}(1-0)$ emission in greyscale, overlaid by the ${ }^{12} \mathrm{CO}(1-0)$ velocity field. The white and black lines are for positive and negative velocity relative to the reference velocity at $2400 \mathrm{~km} \mathrm{~s}^{-1}$.

semi-major axis ( $\sim 6.5 \mathrm{kpc})$. Several studies (e.g., Athanassoula 1992; Combes \& Elmegreen 1993; Sempere et al. 1995) have shown that the ratio of the bar corotation radius to the bar semimajor axis length is in the range 1-1.5. The determination of the bar pattern speed is not strongly dependent on the exact value of this ratio since the frequency $\Omega$ is flat in that domain. The bar pattern speed $\Omega_{\text {bar }}$ is $\sim 22 \mathrm{~km} \mathrm{~s}^{-1} \mathrm{kpc}^{-1}$ with a corotation (CR) at a radius of $40^{\prime \prime}(6.5 \mathrm{kpc})$, using a value of 1 for the ratio of the radius of the $\mathrm{CR}$ to the length of the bar semi-major axis. The inner Lindblad resonances (ILRs), if they exist, are the loci where the bar pattern speed equals $\Omega-\kappa / 2$ (Fig. 11). Thus, the epicyclic approximation suggests that two ILRs are present near the center. The rough radii for the inner and outer ILRs (iILR and oILR) are $6{ }^{\prime \prime}$ and $10^{\prime \prime}$ (1 and $1.7 \mathrm{kpc}$ ). 4:1 resonances, or (inner/outer) ultraharmonic resonances (i/oUHR), with $\Omega_{\mathrm{bar}}=\Omega \pm \kappa / 4$, are found at radii of $24^{\prime \prime}$ (3.9 kpc; iUHR) and $100^{\prime \prime}(16.5 \mathrm{kpc}$; oUHR). At these resonances the star orbits are periodic and form rings (e.g., Byrd et al. 1998). Contrary to the UHR location, the locations of the ILRs strongly depend on the bar pattern speed. Table 6 summarizes the locations of the resonances found with the application of the epicyclic approximation, valid for asymptotically weak bars. In the presence of an even moderately strong bar, an accurate determination of the resonance positions requires a full dynamical model, together with orbit analysis, which is beyond the scope of this paper.

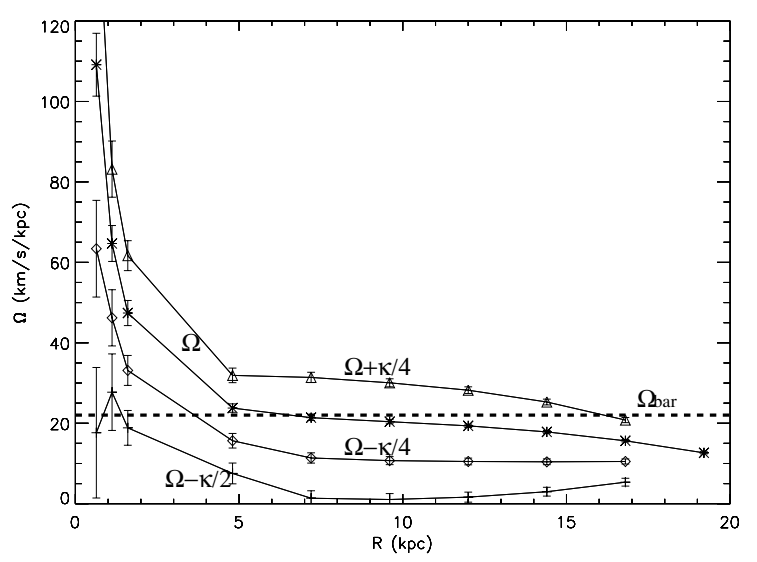

Fig. 11. Azimuthal frequency $\Omega$ (stars) estimated from the rotation curve ( $\mathrm{HI}+\mathrm{CO}$ data). The first three data points are from our new $\mathrm{CO}$ (1-0) observations. The data points at larger radii are from HI observations (Wilcots et al. 2001). The UHR (diamond) and ILR (cross) locations have been estimated by setting the bar corotation radius to the semi-major axis radius of the bar $\left(\sim 40^{\prime \prime}\right)$. The bar pattern speed is estimated to be $22 \mathrm{~km} \mathrm{~s}^{-1} \mathrm{kpc}^{-1}$.

Table 6. Dynamical resonances as estimated from a rough epicyclic model for NGC 6764. $\Omega_{\mathrm{bar}}=22 \mathrm{~km} \mathrm{~s}^{-1} \mathrm{kpc}^{-1}$. See the text for a detailed description.

\begin{tabular}{lll}
\hline \hline Resonance & $n$ & Radius \\
& $\left(\Omega_{\text {bar }}=\Omega+n \times \kappa\right)$ & \\
\hline iILR & $-1 / 2$ & $6 \pm 6^{\prime \prime}$ \\
oILR & $-1 / 2$ & $10 \pm 10^{\prime \prime}$ \\
iUHR & $-1 / 4$ & $24 \pm 5^{\prime \prime}$ \\
CR & 0 & $40 \pm 12^{\prime \prime}$ \\
oUHR & $+1 / 4$ & $100 \pm 10^{\prime \prime}$ \\
\hline
\end{tabular}

\subsubsection{Non-circular motions}

Figure 12 shows the position-velocity (PV) diagrams of the ${ }^{12} \mathrm{CO}(1-0)$ and ${ }^{12} \mathrm{CO}(2-1)$ emission along the major and minor axes $\left(\mathrm{PA}=62^{\circ}\right.$ and $\left.\mathrm{PA}=152^{\circ}\right)$ of $\mathrm{NGC} 6764$, along with a PV diagram at $\mathrm{PA}=90^{\circ}$ for comparison with the ${ }^{12} \mathrm{CO}(1-0)$ observations made with the Nobeyama Millimeter Array (Eckart et al. 1996). As already shown by Eckart et al. (1996), the PV diagrams imply an asymmetric distribution of molecular gas near the nucleus. The $\mathrm{CO}$ intensity is higher for gas at redshifted velocities, with respect to the systemic velocity, i.e., on the eastern side. The PV diagram at PA $=90^{\circ}$ in the ${ }^{12} \mathrm{CO}(1-0)$ emission shows strong streaming motion at $x=-4^{\prime \prime}$, which is located slightly inside the iILR $(r \sim$ $\left.6^{\prime \prime}\right)$. The ${ }^{12} \mathrm{CO}(2-1)$ PV diagram along the minor axis reveals large streaming motions at $x=1^{\prime \prime}-2^{\prime \prime}$, with a velocity near $-200 \mathrm{~km} \mathrm{~s}^{-1}$. Similarly, the ${ }^{12} \mathrm{CO}(1-0) \mathrm{PV}$ diagram along the minor axis shows an area of lower density gas on the eastern side at radii $<5^{\prime \prime}$, with streaming motions at negative velocities. The spur visible between $x$-positions $1^{\prime \prime}$ and $6^{\prime \prime}$ in the velocity range $[-30,80] \mathrm{km} \mathrm{s}^{-1}$ (relative to $2400 \mathrm{~km} \mathrm{~s}^{-1}$ ) is located in the molecular outflow, and is consistent with the ${ }^{12} \mathrm{CO}(1-0)$ spectrum integrated over the outflow area. Assuming a trailing spiral structure, the northern part of NGC 6764 is closer to us, which implies largely redshifted (positive) velocities for the radial outflow in the northern side, as is seen in the PV diagram and in the integrated ${ }^{12} \mathrm{CO}(2-1)$ spectrum. 

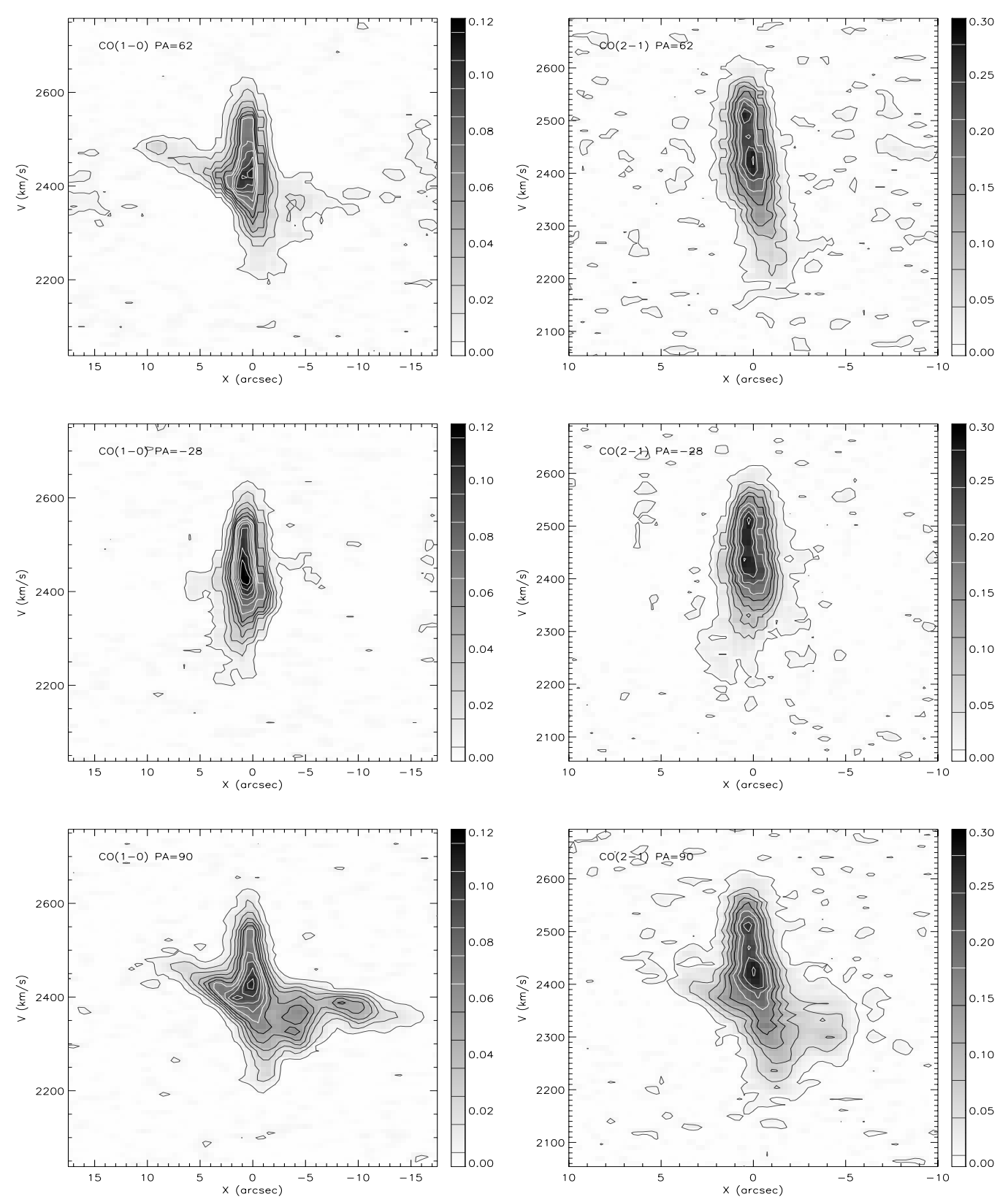

Fig. 12. Position-velocity diagram of the ${ }^{12} \mathrm{CO}(1-0)$ (left) and ${ }^{12} \mathrm{CO}(2-1)$ (right) emission in the center of NGC 6764. The zero-offset is at the CO peak. The major axis PA is $62^{\circ}$, and the PA of $90^{\circ}$ has been included to compare with Fig. 1 of Eckart et al. (1996). The units are in Jy beam ${ }^{-1}$.

\subsection{CO line ratio}

The line ratio for the integrated $\mathrm{CO}$ intensity between the ${ }^{12} \mathrm{CO}(2-1)$ and ${ }^{12} \mathrm{CO}(1-0)$ transitions, $\mathfrak{R}=$ $I\left({ }^{12} \mathrm{CO}(2-1)\right) / I\left({ }^{12} \mathrm{CO}(1-0)\right)$, was computed matching the $\mathrm{u}-\mathrm{v}$ coverage of both transitions and using the same spatial resolution and taking only the ${ }^{12} \mathrm{CO}(1-0)$ and ${ }^{12} \mathrm{CO}(2-1)$ data above a threshold of $3 \sigma$. The result is shown in Fig. 13. The maximum $\mathfrak{R}$ is 0.98 , in agreement with that estimated by Eckart et al. (1991) from single dish observations. Eckart et al. (1991) interpreted the global ratio to arise from moderately warm, dense, and optically thick gas.

To estimate the uncertainty in the line ratio introduced by the missing flux, we compared the $\mathrm{CO}$ line ratio map derived from the single-dish data with the ratio derived from our new PdBI observations. We derive larger values of the ratio at large radii, owing to the lower spatial resolution $\left(\sim 20^{\prime \prime}\right)$ of Eckart et al. (1991). Their maximum value at the center is lower than ours by $\sim 10 \%$. The maximum line ratio $\mathfrak{R}_{\max }$ in each channel (10 $\mathrm{km} \mathrm{s}^{-1}$ width) is shown in Fig. 14. A bimodal feature is clearly seen, with peaks towards the blue and red sides. The blue side with a peak at $-140 \mathrm{~km} \mathrm{~s}^{-1}$ is associated with molecular gas closer to the radio continuum peak, as shown in the CO line ratio channel maps in Fig. 15. At the peak the ratio reaches values of 2-2.5. The red side of the peak at $140 \mathrm{~km} \mathrm{~s}^{-1}$ has a maximum value of about 1.3. This high $\mathrm{CO}$ line ratio in fact applies to the bulk of the molecular gas, as is shown by the $\mathrm{CO}$ line ratio channel maps below.

The ${ }^{12} \mathrm{CO}(2-1) /{ }^{12} \mathrm{CO}(1-0)$ line ratio has been computed for each channel $\left(10 \mathrm{~km} \mathrm{~s}^{-1}\right.$ width). Figure 15 shows the $\mathrm{CO}$ line ratio from a channel at $-180 \mathrm{~km} \mathrm{~s}^{-1}$ up to a channel at $200 \mathrm{~km} \mathrm{~s}^{-1}$ (the systemic velocity of NGC 6764 is $2400 \mathrm{~km} \mathrm{~s}^{-1}$, 


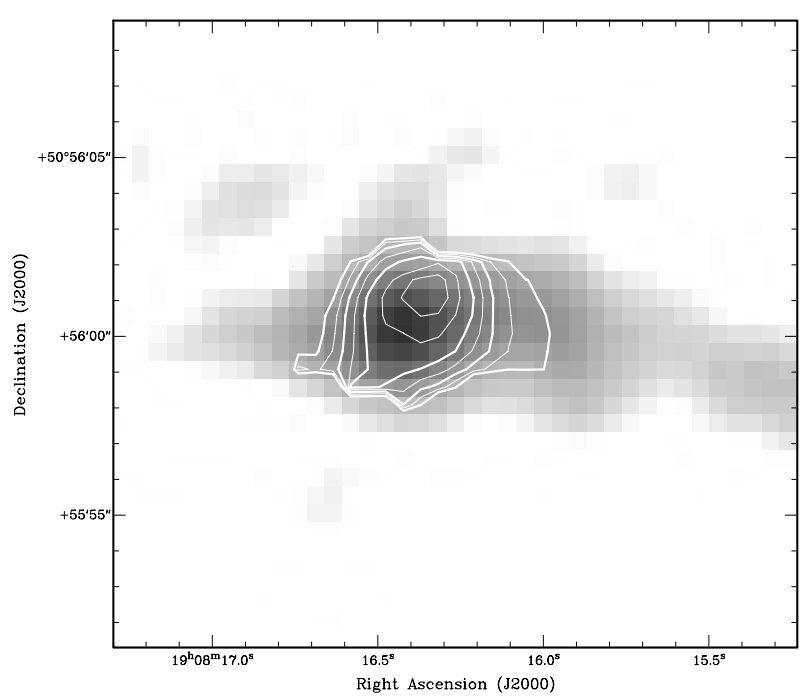

Fig. 13. Integrated intensity ${ }^{12} \mathrm{CO}(1-0)$ line (greyscale) overlaid by contours showing the ${ }^{12} \mathrm{CO}(2-1)$ to ${ }^{12} \mathrm{CO}(1-0)$ line ratio, integrated over all channels. The contours are $0.4,0.5,0.6,0.7,0.8,0.9$ and 0.95 .

corresponding to a zero velocity in the channel maps). From -180 to $-40 \mathrm{~km} \mathrm{~s}^{-1}$ the peak of the CO line ratio is spatially coincident with the nucleus (or the radio continuum peak, as indicated by a cross in the figure). The peak at $-140 \mathrm{~km} \mathrm{~s}^{-1}$ seen in Fig. 14 is likely to be an edge effect. The "true" maximum is reached at $-130 \mathrm{~km} \mathrm{~s}^{-1}$, where the ratio reaches a value of 1.4 at the exact position of the radio continuum peak (to within a pixel width). The western $\mathrm{CO}$ emission feature has a mean CO line ratio of 0.3 , which is likely to be an underestimate because of the filtering out of the extended emission.

The high CO line ratios for the blueshifted gas in the nucleus may be associated with outflowing molecular gas powered by the AGN or the starburst. HSO6 reported a possible blueshifted $\mathrm{HI}$ absorption at $\sim 120 \mathrm{~km} \mathrm{~s}^{-1}$ against the compact core which may be related to the molecular gas with such high CO line ratios. We note that the radio continuum map (Fig. 1) has hints of a nuclear radio jet towards the south, likely associated with blueshifted motions there as expected if the orientation of NGC 6764 with respect to the line of sight is taken into account. A redshifted counterpart with such a high line ratio is not detected.

\section{4. $\mathrm{H}_{2}$ mass}

Adopting a standard conversion factor of $X_{\mathrm{CO}}=2.3 \times 10^{20} \mathrm{~cm}^{-2}$ $\left(\mathrm{K} \mathrm{km} \mathrm{s}^{-1}\right)^{-1}$ (e.g., Strong et al. 1988), the total mass of molecular hydrogen $\left(\mathrm{H}_{2}\right)$ is:

$M_{\mathrm{H}_{2}}=9 \times 10^{3} S_{\mathrm{CO}} D^{2}$

where $S_{\mathrm{CO}}$ is the integrated CO flux density in $\mathrm{Jy} \mathrm{km} \mathrm{s}^{-1}$ and $D$ is the distance in Mpc. The integrated ${ }^{12} \mathrm{CO}(1-0)$ flux density from our new map gives a total molecular hydrogen mass of $1.0 \times 10^{9} \mathcal{M}_{\odot}$. It does not take into account the $50 \%$ of flux filtered out by the interferometer observations.

In the Milky Way's disk the $\mathrm{X}_{\mathrm{CO}}$ conversion factor derived from observations has been found to match the theoretical conversion factor quite well (e.g., Radford et al. 1991). However, there is growing evidence that the conversion factor is overestimated by a factor of 3-10 in the central part of galaxies, especially in those with nuclear starbursts (Downes \& Solomon 1998). The physical reasons for this discrepancy have been discussed in several papers, and include the metallicity gradient

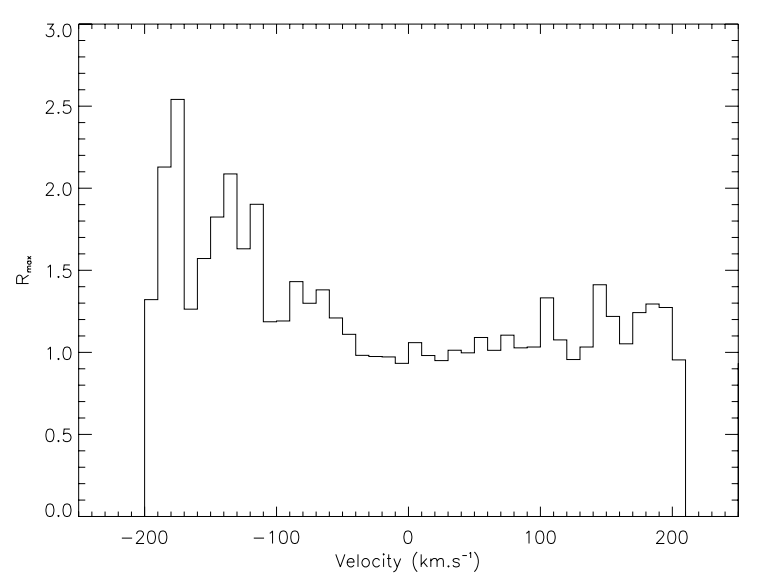

Fig. 14. Maximum ${ }^{12} \mathrm{CO}(2-1)$ to ${ }^{12} \mathrm{CO}(1-0)$ line ratio $\mathfrak{R}_{\max }$ computed in the center of NGC 6764 for each ${ }^{12} \mathrm{CO}(1-0)$ channel $\left(10 \mathrm{~km} \mathrm{~s}^{-1}\right.$ width).

(e.g., Dahmen et al. 1998), broadening of the CO line by the bulge potential (e.g., Stacy et al. 1989; Mauersberger et al. 1996), and changes in the physical conditions of the circumnuclear gas (e.g., its optical depth, temperature, and density). In NGC 253, a nearby starburst galaxy, Mauersberger et al. (1996) found a conversion factor $\sim 7$ times lower than $X_{\mathrm{CO}}^{\text {std }}$ in the central part of the galaxy. They argued that the central potential well is broadening the $\mathrm{CO}$ lines, and its emission could be partially optically thin. The conversion factor has been found to be 3 to 10 times lower in the galactic bulges compared to their disks (Sodroski et al. 1995). Recently, Weiß et al. (2001) analyzed the variations of $X_{\mathrm{CO}}$ in the starburst of M 82 in detail. They found that " $X_{\mathrm{CO}}$ is a function of the intrinsic gas properties, which strongly depend on environmental effects" (massive star formation). This leads to a variation of $X$ by a factor of 5-10, compared to the Galactic value of $X_{\mathrm{CO}}=1.6 \times 10^{20} \mathrm{~cm}^{-2} \mathrm{~K} \mathrm{~km} \mathrm{~s}^{-1}$.

The dust mass computed from the IRAS fluxes at 60 and $100 \mu \mathrm{m}$ gives a total mass of $M_{\mathrm{d}}=1.4 \times 10^{6} \mathcal{M}_{\odot}$ with a mean temperature of $36.5 \mathrm{~K}$. It is known from FIR observations with ISO that the IRAS data are missing a cold dust component that emits beyond $150 \mu \mathrm{m}$ and has a temperature of $T_{\mathrm{d}}<20 \mathrm{~K}$, (Alton et al. 1998; Trewhella et al. 2000). This component can be 2-10 times more massive than the warm dust component.

In NGC 6764 the gas-to-dust mass ratio, using the standard CO-to- $\mathrm{H}_{2}$ conversion factor and correcting for the missing flux in the interferometer map, leads to $M_{\mathrm{H}_{2}} / M_{\text {dust }} \sim 1500 \pm 225$. This factor is much larger than the typical value found in normal spiral galaxies (an average ratio of $\sim 700$; Leon et al. 1998) using IRAS-derived dust masses. This indicates that the molecular gas mass obtained from the ${ }^{12} \mathrm{CO}(1-0)$ luminosity and the standard conversion factor has most likely been overestimated.

The high gas-to-dust mass ratio means that the $\mathrm{H}_{2}$ mass has probably been overestimated by a factor of 2-3 in the center of NGC 6764, when using the standard conversion factor $X \sim X_{\mathrm{CO}}^{\text {std }}$. This overestimate may be even more severe in the nucleus because of the intense starburst. Thus we estimate the total $\mathrm{H}_{2}$ mass to be $7 \times 10^{8} \mathcal{M}_{\odot}$, taking into account the filtered out flux in the ${ }^{12} \mathrm{CO}(1-0)$ interferometric map.

\section{Outflow}

The basic morphological parameters for the outflow from the $20 \mathrm{~cm}$ radio continuum observations are: a projected base of 

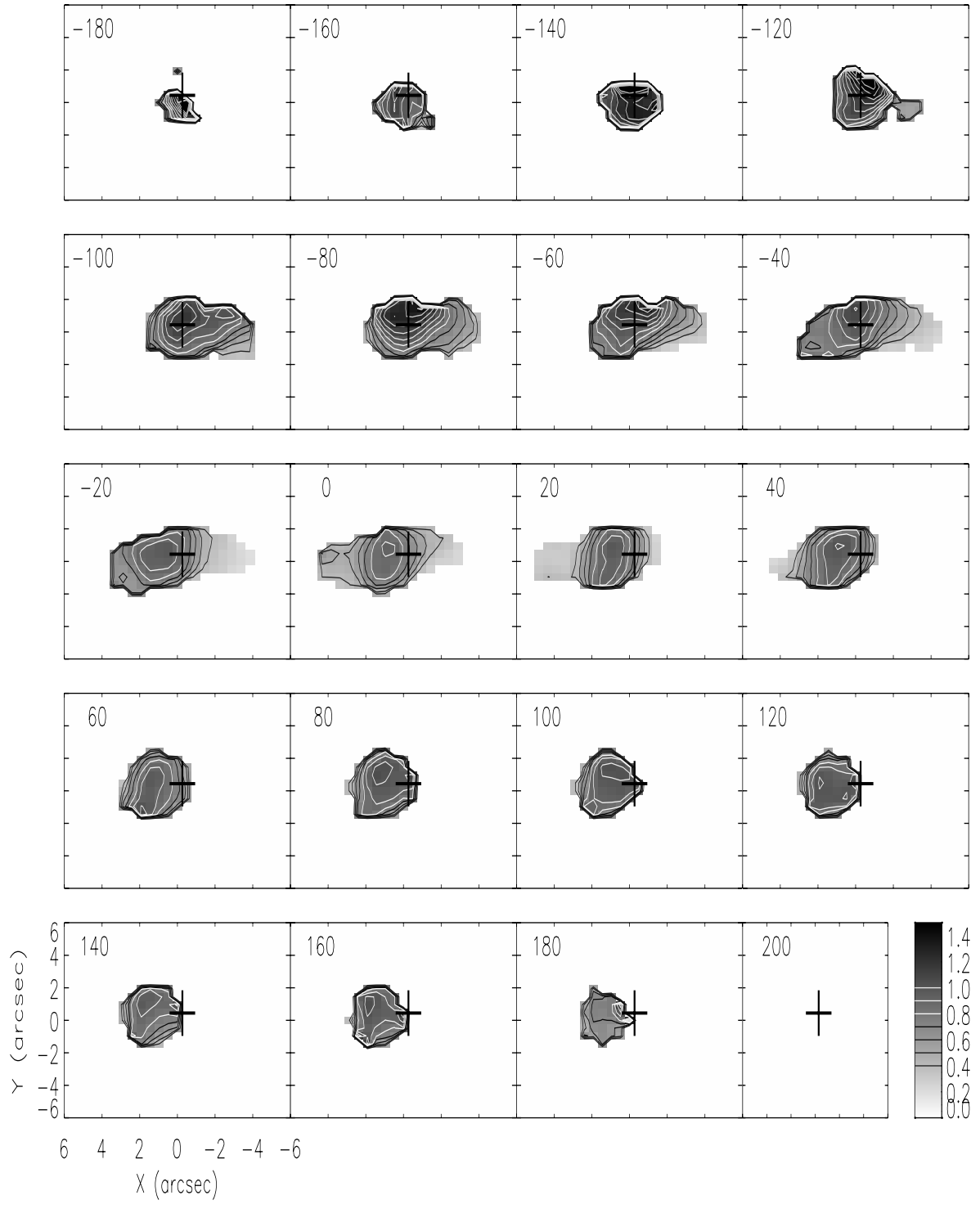

Fig. 15. ${ }^{12} \mathrm{CO}(2-1)$ to ${ }^{12} \mathrm{CO}(1-0)$ line ratio for different velocities, as indicated in each panel, with a channel width of $10 \mathrm{~km} \mathrm{~s}^{-1}$. The first contour is at 0.4 and the contours increase in steps of 0.1 . The radio continuum peak is shown by a cross. radius $3.5^{\prime \prime}(540 \mathrm{pc})$ and a projected height of $7^{\prime \prime}$ (1080 pc) above the galaxy plane. The mass-load in the outflow is estimated from the spatial distribution of the ${ }^{12} \mathrm{CO}(1-0)$ emission. The ${ }^{12} \mathrm{CO}(1-0)$ emission was integrated in the area shown in Fig. 2 only for the northern part of the outflow. The area was chosen in order to avoid the contamination from the molecular gas emission in the disk, given the inclination of the galaxy and the spatial resolution of the observations. The ${ }^{12} \mathrm{CO}(1-0)$ integrated intensity in the out-of-plane outflow is $1.4 \mathrm{Jy} \mathrm{km} \mathrm{s}^{-1}$.

The observed energy of the different gas phases in the outflow is an indicator of the physical processes responsible for the outflow. Assuming that the bulk of the molecular gas is perpendicular to the disk plane, the expansion velocity $V_{\exp }$ is estimated from the spur in ${ }^{12} \mathrm{CO}(1-0)$ seen between -30 and $80 \mathrm{~km} \mathrm{~s}^{-1}$ on the PV diagram (see Fig. ). We take a projected expansion velocity of $25 \mathrm{~km} \mathrm{~s}^{-1}$, which gives an expansion velocity of $53 \mathrm{~km} \mathrm{~s}^{-1}$. This value is quite uncertain since we know already that some molecular gas is reaching an expansion velocity of $170 \mathrm{~km} \mathrm{~s}^{-1}$. Applying a correction of $1 / 3$ to the CO-to- $\mathrm{H}_{2}$ conversion factor (see Sect. 4.4) the total molecular gas mass in the outflow is $4.3 \times 10^{6} \mathcal{M}_{\odot}$. Thus the molecular gas kinetic energy $E_{\text {kin }}$ in the outflow is $E_{\text {kin }}=\frac{1}{2} M_{\text {gas }} * V_{\exp }^{2}=2.4 \times 10^{53} \mathrm{erg}$.
For a starburst with a $S F R=1 \mathcal{M}_{\odot} / y r$, different star formation models have been computed (instantaneous or continuous). The total energy released by SNs and stellar wind at the age of the recent NGC 6764 starburst ( $~ 5 \mathrm{Myr}$ ) is in the range of $10^{54}-10^{55} \mathrm{erg}$. Thus there is no need to invoke AGN or other mechanisms, such as hypernovae or tidal interaction, to drive the outflow, even if the AGN seems to have an effect on the molecular gas physical conditions (see Sect. 4.3) and the data cannot exclude the AGN from being the major energy source either.

To further study the energy budget, we computed the total thermal energy $E_{\mathrm{x}}$ in the hot X-ray gas, which was determined by the HRI ROSAT instrument. The X-ray emission is composed by two components: one varying on the timescale of several days and a more diffuse one which extends up to $15^{\prime \prime}$ from the nucleus, roughly along the minor axis (Schinnerer et al. 2000). We make use of the X-ray parameters derived by Eckart et al. (1996) from the ROSAT observations in the $0.1-2.4 \mathrm{keV}$ band. They estimated an electron density $n_{\mathrm{e}} \sim 0.2 f_{\mathrm{s}}^{-1 / 2} \mathrm{~cm}^{-3}$, where $f_{\mathrm{s}}$ is the volume filling factor, the volume of the extended X-ray gas as $V \sim 7 \times 10^{63} \mathrm{~cm}^{3}$, and the temperature of the $\mathrm{X}$-ray gas as $T \sim 4 \times 10^{6} \mathrm{~K}$. The total thermal energy is then given by $E_{\mathrm{x}} \sim 3 n_{\mathrm{e}} k T V f_{\mathrm{s}}$, i.e. $E_{\mathrm{x}} \sim 2.3 \times 10^{54} f_{\mathrm{s}}^{1 / 2}$ erg. We 
use $f_{\mathrm{s}}=0.01$, as was found for the starburst galaxy NGC 3256 (Heckman et al. 1990; Moran et al. 1999). Thus $E_{\mathrm{x}}$ is estimated to be $\sim 2.3 \times 10^{53} \mathrm{erg}$. The current starburst in NGC 6764 appears to be capable of releasing sufficient energy to power the hot X-ray gas bubble.

To estimate the distribution of the radio continuum emission at $20 \mathrm{~cm}$ in the outflow bubble, the nuclear component has been subtracted by fitting a Gaussian to the nuclear flux above a threshold of $4 \mathrm{mJy}$ beam $^{-1}$. The total flux remaining in the center-subtracted map is $63 \mathrm{mJy}$, or about $60 \%$ of the total seen in the VLA observation. There is an offset of the centroid of the outflow emission relative to the position of the nuclear radio continuum peak, and relative to the disk plane. This offset can be due to various reasons: a) the starburst is located in the western part of the nucleus, and slightly below the plane, as suggested by the lower gas column density south of the nucleus; b) there is a possible contribution from a radio jet in the southern part in addition to the starburst-induced outflow.

\section{Discussion}

We compare the $\mathrm{CO}$ and $\mathrm{H} \alpha$ observations to the different hydrodynamical models of Tomisaka \& Ikeuchi (1988) performed for a smooth gas component with various scenarios of starburst. The case D of Tomisaka \& Ikeuchi (1988) has a starburst with a supernova rate comparable to the one found in NGC 6764 $\left(10^{-2} \mathrm{yr}^{-1}\right.$ : the bubble reaches $z \sim 1.2 \mathrm{kpc}$ after $9 \mathrm{Myr}$, which is difficult to conciliate with the W-R star age and population. In the case $\mathrm{C}$, with an ambient density of $n \approx 100 \mathrm{~cm}^{-3}$, the height of the bubble reaches $1 \mathrm{kpc}$ after 7 Myr. Moreover they performed a simulation with a short "explosion" which drives the bubble height to $z \approx 1.3 \mathrm{kpc}$ as fast as in $1 \mathrm{Myr}$. From all these different models, we can conclude that the recent starburst has been intense and short 3-7 Myr ago, in complete agreement with the stellar population analysis of Schinnerer et al. (2000). From the comparison with these simulations we can conclude that the hot gas in NGC 6764 is still in a confined bubble, and has not yet escaped to the halo.

The NSB in NGC 6764 is very similar to the NSB in NGC 2782 (Jogee et al. 1998, 1999). However, NGC 2782 has no nuclear activity, apart from the starburst. The $\mathrm{CO}$ spurs in NGC 2782 have a total molecular gas mass of $2 \times 10^{7} \mathcal{M}_{\odot}$, a total molecular gas kinetic energy of $\sim 8 \times 10^{53} \mathrm{erg}$, and a thermal energy of $\sim 2 \times 10^{56} f^{0.5}$. These numbers are larger by factors of 5-10 compared to the energy released in NGC 6764. Nevertheless the size of the outflows is comparable. This is probably due to the lower ISM density in NGC 6764 than in NGC 2782.

Observations (Seaquist \& Clark 2001; Walter et al. 2002) of ${ }^{12} \mathrm{CO}(3-2)$ and ${ }^{12} \mathrm{CO}(1-0)$ emission in the prototypical starburst galaxy M 82 indicate a large amount of molecular gas ( $>3 \times 10^{8} \mathcal{M}_{\odot}$ in the outflow with a maximum outflow velocity of $230 \mathrm{~km} \mathrm{~s}^{-1}$ and an estimated kinetic energy of $\sim 10^{55} \mathrm{erg}$. Similarly to our estimate, Weiß et al. (2001) found a CO-to- $\mathrm{H}_{2}$ conversion 3 times lower than the Galactic conversion factor. The height of the molecular gas outflow is about $1 \mathrm{kpc}$ above the plane with an estimated starburst age of $10 \mathrm{Myr}$. The comparison with NGC 6764 indicates that in this galaxy the build-up of the molecular outflow was faster by a factor $2-3$. The main difference is the molecular gas mass involved in the outflow, which is an order of magnitude larger in M 82 than in NGC 6764.

HS06 present a compilation of 10 galaxies with non-thermal bubbles that shows that all are associated with an AGN. They link the origin of these bubbles to the presence of an AGN. We note that 6 galaxies of their sample $(60 \%)$ have a FIR luminosity larger than the one of NGC 6764, if we adopt their luminosities. The presence of an AGN may not be sufficient to create these bubbles. It may indicate that the association of a starburst, present or recent, with an AGN could be the key to generate nonthermal radio bubbles. The creation of such feature would depend on a short time scale combination of the two processes: the starburst and the AGN. Nevertheless we note that the presence of radio AGN is very low in spiral galaxies (J. Sabater, private communication) which makes the correlation between radio-AGN and non-thermal bubble very significant. Thus a statistical study with a large sample of galaxy with non-thermal bubbles would help to disentangle their origins.

NGC 6764, like other composite galaxies with a nuclear starburst and an AGN, shows that the molecular gas evolution in the very center is mainly driven by the starburst, especially in the molecular outflow. Even if the AGN has an active role in the central evolution, as shown by the gas chemistry in the Seyfert 2 galaxy NGC 1068 (Usero et al. 2004), the only possible influences of the AGN on the molecular gas, at the resolution of the observations of the CO emission in NGC 6764, appears to be the high $\mathrm{CO}$ line ratio in the very center, and likely the high HCN/CO ratio already found by Kohno et al. (2001). No clear dynamic signature is observed. The AGN are able to inject vast amounts of energy into their host galaxies and should have a disruptive influence on the molecular gas in the central regions.

\section{Summary}

We have found the following results from our high-resolution mapping of the ${ }^{12} \mathrm{CO}(1-0)$ and ${ }^{12} \mathrm{CO}(2-1)$ millimetre lines and 3.5 and $20 \mathrm{~cm}$ radio continuum emission in the circumnuclear area of the NSB galaxy NGC 6764.

1. The bulk of the ${ }^{12} \mathrm{CO}$ emission is concentrated in the very center of NGC 6764 and is offset from the $20 \mathrm{~cm}$ radio continuum peak by $\sim 1^{\prime \prime} .2$ (with respect to the ${ }^{12} \mathrm{CO}(1-0)$ emission peak). A more extended component is detected along the bar on the western side of the nucleus.

2. Non-thermal radio continuum emission at 3.5 and $20 \mathrm{~cm}$ is observed above and below the galactic plane in a nuclear bubble-like outflow, as already shown by HS06 and Laine et al. (2006). This outflow is detected out to $z=1.3 \mathrm{kpc}$ above the galactic plane (deprojected). ${ }^{12} \mathrm{CO}(1-0)$ emission is detected at the NE boundary of the outflow. About $4.3 \times$ $10^{6} \mathcal{M}_{\odot}$ of molecular gas is driven by the outflow out of the plane of the galaxy.

3. The total molecular gas mass is $6.7 \times 10^{8} \mathcal{M}_{\odot}$ using a CO-to- $\mathrm{H}_{2}$ conversion factor equal to $1 / 3$ of the Galactic value. The use of such a value is motivated by the large gasto-dust mass ratio and previous studies of molecular gas in starburst galaxies.

4. The ${ }^{12} \mathrm{CO}(2-1) /{ }^{12} \mathrm{CO}(1-0)$ line ratio was computed in each channel $\left(10 \mathrm{~km} \mathrm{~s}^{-1}\right.$ velocity width). It reaches a maximum of $1-2$ at the nucleus, and is likely associated with the nuclear AGN (it coincides with the $20 \mathrm{~cm}$ radio continuum peak). In the bulk of the molecular gas the maximum ratio is about $1-1.3$, consistent with warm $\left(T_{\text {kin }} \geq 20 \mathrm{~K}\right)$ and dense gas $\left(n_{\mathrm{H}_{2}}>2 \times 10^{4} \mathrm{~cm}^{-3}\right)$, as found by Eckart et al. (1991).

5. An important fraction of the radio continuum at $3.5 \mathrm{~cm}$ is thermal. The outflow morphology and the ISM properties indicate an age of 3-7 Myr for the recent starburst, by comparing the observations to hydrodynamical simulations. It appears that the outflow is still confined and not freely 
expanding. A "hot-spot" of thermal gas with a flat spectral index $(\sim-0.2)$ is located near the northern boundary of the bubble/outflow.

6. The kinetic and thermal energies of the molecular and hot gas components in the bubble can be fully accounted for by the energy released from the NSB, even if the data cannot exclude the AGN from being the major energy source.

Acknowledgements. We thank the anonymous referee for a careful reading and very detailed report which helped to improve this paper significantly. This research has made use of the NASA/IPAC Extragalactic Database (NED) which is operated by the Jet Propulsion Laboratory, California Institute of Technology, under contract with the National Aeronautics and Space Administration. During part of this research S. Leon was supported by a Marie Curie Individual Fellowship contract HPMF-CT-20002-01734 from the European Union. We would like to thank David Frayer and Eric Wilcots for providing their $\mathrm{H} \alpha$ and HI data, respectively.

\section{References}

Alton, P. B., Bianchi, S., Rand, R. J., et al. 1998, ApJ, 507, 125

Athanassoula, E. 1992, MNRAS, 259, 345

Baum, S. A., O’Dea, C. P., Dallacassa, D., de Bruyn, A. G., \& Pedlar, A. 1993, ApJ, 419, 553

Byrd, G. G., Ousley, D., \& dalla Piazza, C. 1998, MNRAS, 298, 78

Caplan, H., \& Deharveng, L. 1986, A\&A, 155, 297

Colina, L., Arribas, S., \& Borne, K. D. 1999, ApJ, 527, L13

Combes, F. 2001, The Central Kiloparsec of Starbursts and AGN: The La Palma Connection, ed. J. H. Knapen, J. E. Beckman, I. Shlosman, \& T. J. Mahoney (ASP) 249, 475

Combes, F., \& Gerin, M. 1985, A\&A, 150, 327

Combes, F., \& Elmegreen, B. G. 1993, A\&A, 271, 391

Condon, J. J., \& Yin, Q. F. 1990, ApJ, 357, 97

Dahmen, G., Huttemeister, S., Wilson, T. L., \& Mauersberger, R. 1998, A\&A, 31,959

Dopita, M. A. 1985, ApJ, 295, 5

Downes, D., \& Solomon, P. M. 1998, ApJ, 507, 615

Eckart, A., Cameron, M., Jackson, J. M., et al. 1991, ApJ, 372, 67

Eckart, A., Cameron, M., Boller, Th., et al. 1996, ApJ, 472, 588

Franceschini, A., Silva, L., Fasano, G., et al. 1998, ApJ, 506, 600

Hota, A., \& Saikia, D. J. 2006, MNRAS, 371, 945 (HS06)

Heckman, T. M., Armus, L., \& Miley, G. K. 1990, ApJS, 74, 833

Hill, T. L., Heisler, C. A., Norris, R. P., Reynolds, J. E., \& Hunstead, R. W. 2001, AJ, 121, 128

Hummel, E., van Gorkom, J. H., \& Kotanyi, C. G. 1983, ApJ, 267, 5

Hummel, E., Beck, R., \& Dettmar, R.-J. 1991, A\&AS, 87, 309

Irwin, J. A., \& Sofue, Y. 1996, ApJ, 464, 738
Jogee, S., Kenney, J. D. P., \& Smith, B. J. 1998, ApJ, 494, 185 Jogee, S., Kenney, J. D. P., \& Smith, B. J. 1999, ApJ, 526, 665 Kenney, J. D. P., Carlstrom, J. E., \& Young, J. S. 1993, ApJ, 418, 687

Kennicutt, R. C., Jr. 1998, ApJ, 498, 541

Kohno, K., et al. 2001, The Central Kiloparsec of Starbursts and AGN: The La Palma Connection, ed. J. H. Knapen, J. E. Beckman, J. E., Shlosman, \& I. Mahoney (San Francisco: ASP), ASP Conf. Proc., 249, 672

Kunth, D. Legrand, F. Tenorio-Tagle, G., et al. 2002, Ap\&SS, 281, 261

Krolik, J. H., \& Begelman, M. C. 1986, 308, L55

Laine, S., Shlosman, I., Knapen, J. H., \& Peletier, R. F. 2002, ApJ, 567, 97

Laine, S., Kotilainen, J. K., Reunanen, J., Ryder, S. D., \& Beck, R. 2006, AJ, 131,701

Launhardt, R., Zylka, R., \& Mezger, P. G. 2002, A\&A, 384, 112

Lee, S.-W., Irwin, J. A., Dettmar, R.-J., et al. 2001, A\&A, 377, 759

Leon, S., Combes, F., \& Menon, T. K. 1998, A\&A, 330, 37

Leon, S., Meylan, G., \& Combes, F. 2000, A\&A, 359, 907

Mauersberger, R., Henkel, C., Wielebinski, R., Wiklind, T., \& Reuter, H.-P. 1996, A\&A, 305, 421

Mihos, J. C., \& Hernquist, L. 1994, ApJ, 425, 13

Moran, E. C., Lehnert, M. D., \& Helfand, D. J. 1999, ApJ, 526, 649

Norman, C. A., \& Ikeuchi, S. 1989, ApJ, 345, 372

Osterbrock, D. E., \& Cohen, R. E. 1982, ApJ, 261, 640

Radford, S. J. E., Downes, D., \& Solomon, P. M. 1991, ApJ, 368L, 15

Rozas, M., Beckman, J. E., \& Knapen, J. H. 1996, A\&A, 307, 735

Sakamoto, K., Ho, P. T. P., Iono, D., et al. 2006, ApJ, 636, 685

Sanders, D. B., \& Mirabel, I. F. 1985, ApJ, 298, 31

Scalo, J., \& Chappell, D. 1999, MNRAS, 310, 1

Scoville, N. Z., Yun, M. S., Sanders, D. B., Clemens, D. P., \& Waller, W. H. 1987, ApJS, 63, 821

Schinnerer, E., Eckart, A., \& Boller, Th. 2000, ApJ, 545, 205

Seaquist, E. R., \& Clark, J. 2001, ApJ, 552, 133

Sempere, M. J., Garcia-Burillo, S., Combes, F., \& Knapen, J. H. 1995, A\&A, 296,45

Sodroski, T. J., Odagar, N., Swek, E., et al. 1995, ApJ, 452, 262

Stacy, J. G., Bitran, M. E., Dame, T. M., \& Thaddeus, P. 1989, The Center of the Galaxy: Proceedings of the 136th Symp. IAU, Los Angeles, USA, ed. M. Morris (Dordrecht: Kluwer Academic Publishers), 157

Strickland, D. K., Ponman, T. J., \& Stevens, I. R. 1997, A\&A, 320, 378

Strong, A. W., Bloemen, J. B. G. M., et al. 1988, A\&A, 207, 1

Taylor, D., Dyson, J. E., \& Axon, D. J. 1992, MNRAS, 255, 351

Tomisaka, K., \& Ikeuchi, S. 1988, ApJ, 330, 695

Trewhella, M., Davies, J. I., Alton, P. B., Bianchi, S., \& Madore, B. F. 2000, ApJ, 543,153

Usero, A., García-Burillo, S., Fuente, A., Martín-Pintado, J., \& Rodríguez-Fernández, N. J. 2004, A\&A, 419, 897

Walter, F.,Weiss, A., \& Scoville, N. Z. 2002, ApJ, 580, 21

Weiß, A., Neininger, N., Hüttemeister, S., \& Klein, U. 2001, A\&A, 365, 571

White, R. L., \& Becker, R. H. 1992, ApJS, 79, 331

Zurita, A., Rozas, M., \& Beckman, J. E. 2000, A\&A, 363, 9 